\title{
Univalent Foundations as Structuralist Foundations
}

\author{
Dimitris Tsementzis*
}

May 4, 2016

\begin{abstract}
The Univalent Foundations of Mathematics (UF) provide not only an entirely non-Cantorian conception of the basic objects of mathematics ("homotopy types" instead of "sets") but also a novel account of how foundations ought to relate to mathematical practice. In this paper, I intend to answer the question: In what way is UF a new foundation of mathematics? I will begin by connecting UF to a pragmatist reading of the structuralist thesis in the philosophy of mathematics, which I will use to define a criterion that a formal system must satisfy if it is to be regarded as a "structuralist foundation." I will explain why neither set-theoretic foundations like ZFC nor category-theoretic foundations like ETCS are structuralist in my sense, essentially because of an undue emphasis on ontology at the expense of language. Then I will argue that UF is better-able to live up to the proposed criterion for a structuralist foundation than any currently available foundational proposal. First, by showing that most criteria of identity in the practice of mathematics can be formalized in terms of the preferred criterion of identity between the basic objects of UF ("homotopy equivalence"). Second, by countering several objections that have been raised against UF's capacity to serve as a foundation for the whole of mathematics.
\end{abstract}

*Department of Philosophy, Princeton University. e-mail: dtsement@princeton.edu I would like to thank (in random order) John Burgess, Paul Benacerraf, Hans Halvorson, Steve Awodey, Mike Shulman, Chris Kapulkin, Vladimir Voevodsky, Colin McLarty, David Corfield, Richard Williamson, Urs Schreiber, Harry Crane, as well as audiences in Philadelphia, London and Princeton. Lastly, I would like to single out in thanks an anonymous referee, who provided extremely detailed and illuminating comments that resulted in many significant improvements. 


\section{Introduction}

In one way or another, structuralism in mathematics is about getting rid of inessential properties of mathematical objects. It doesn't matter whether the number 3 is written as III, iii or $\{\{\{\varnothing\}\}\}$. What matters is that 3 comes before 4 and after 2 . It doesn't matter whether 3 contains $\{\{\varnothing\}\}$ as an element. What matters is that 3 is the immediate successor of the only even prime. Thus, on some level, to be a structuralist about mathematical objects is to believe that the only properties of those objects that matter are those we may call structural properties. Everything else we can say of mathematical objects is nonsense (or, more charitably, non-mathematical).

What could this possibly have to do with the foundations of mathematics? Here is a wild thought: What if the non-structural properties of mathematical objects could be ruled out purely by grammatical considerations? In other words, what if expressing mathematical nonsense like " $\{\{\varnothing\}\} \in 3$ " became a grammatical impossibility rather than a philosophical curiosity? A structuralist foundation for mathematics - as I will use the term - would be a formal system that achieves this, i.e. one in which to express nonsense becomes a grammatical impossibility. In this paper, I will argue that the Univalent Foundations of mathematics provide a foundation that does justice to this idea.

Other than perhaps the early Wittgenstein, the first person to envision such a "nonsense-free" foundation was M. Makkai in his writings on First Order Logic with Dependent Sorts (FOLDS) [25-27]. Needless to say, an important precursor is the work of Lawvere [22] on the Elementary Theory of the Category of Sets (ETCS) which forms a prototypical example of what has come to be known as a "categorical" (or "category-theoretic") foundation of mathematics, as well as observations of Freyd [12] and Blanc [7] on how dependently-typed languages can be used to avoid making non-structural distinctions. ${ }^{1}$ Despite claims made implicitly by Lawvere [22] and explicitly by McLarty $[32,33]$ (among others) to the effect that ETCS-style foundations are compatible with a broadly structuralist view of mathematical objects, there was no attempt to systematically restrict the language of these foundations so that it is able to express only structural properties. In this endeavour, Makkai was undoubtedly the pioneer. ${ }^{2}$

\footnotetext{
${ }^{1}$ ETCS is also called a "structural" set theory, as opposed to an "extensional" or "material" one like ZFC. For accessible comparisons of the two see [23,35].

${ }^{2}$ Although I will not be dealing with Makkai's system directly in this paper, let me mention that in its most recent incarnation in [27] it goes under the name of Type-Theoretic Categorical Foundations of Mathematics (TTCFM). See Marquis [29] for an exposition as well as philosophical defence of Makkai's views on the foundations of mathematics.
} 
Makkai's vision for a "nonsense-free" foundation of mathematics inspired V. Voevodsky to develop his own new foundational proposal going under the name of Univalent Foundations (UF) [48-50,52]: ${ }^{3}$

Univalent Foundations can be seen as a realization of the vision of Michael Makkai [which] was very important for me in my search for a formal language for contemporary mathematics. ([51], p. 2)

In realizing this vision, UF has exploited a fascinating connection between abstract homotopy theory and Martin-Löf Type Theory (MLTT). ${ }^{4}$ The technical development underlying UF has come to be known as Homotopy Type Theory (HoTT) [48]. Very roughly, HoTT consists of the idea that the basic objects of MLTT ("types") can be interpreted as homotopy types ("abstract shapes"). The key feature of HoTT/UF - and what connects it both to structuralism in the philosophy of mathematics and to "nonsense-free" foundations in Makkai's vein - is the so-called axiom of univalence which states, roughly, that isomorphic objects are identical. ${ }^{5}$ Awodey [3] has already made a start in highlighting the connections between UF, univalence and structuralism and in this paper I will sharpen and further reinforce them. In particular, I will argue that UF is better able to do justice to the ideal of a structuralist foundation than any other foundational proposal currently available, at least for a particular way of making precise what this "structuralist ideal" is supposed to be.

And although I will mainly examine UF from this rather localized dialectical perspective, it is worth emphasizing that UF lies at the tip of a much wider historical arc. The dominant narrative in the foundations of mathematics goes as follows: the increasing complexity of mathematical analysis in the 19th century made it imperative to reconstrue all mathematical propositions as logical (i.e. analytic) truths about simple entities - indeed, ideally, as logic itself. And this demand - through Cantor, Frege, Russell, Dedekind, Peano and others - eventually gave rise to set theory and first-order logic as we now understand it together with an all-encompassing foundation of

\footnotetext{
${ }^{3}$ It is important to note, however, that Voevodsky's and Makkai's motivations for their respective projects are very different. Makkai's motivation was at least partly philosophical, aiming to develop a language that encodes a structuralist view of mathematical objects. On the other hand, Voevodsky's work on foundations had primarily a non-philosophical motivation behind it: to develop usable "proof assistants" that mathematicians can use to verify their theorems.

${ }^{4}$ This connection was discovered independently by Voevodsky [49] and by Awodey and Warren $[4,53]$.

${ }^{5} \mathrm{~A}$ more correct way of paraphrasing the axiom of univalence is this: identity is isomorphic to isomorphism. A fully correct way of paraphrasing the axiom of univalence is this: the (canonical) map that sends identities to isomorphisms is an isomorphism.
} 
mathematics in the form of Zermelo-Frankel set theory $(\mathrm{ZF}(\mathrm{C}))$. Following these developments, Kantian views on the synthetic (and a priori) nature of mathematics are more or less dismissed as the inevitable outgrowth of a much too impoverished logic. ${ }^{6}$ A little-known aspect of this story is that at around the same time as Cantor was laying out the groundwork for the developments that would eventually lead us to ZFC, the idea of a synthetic geometry was reborn through the work of figures such as Riemann, Grassmann, Möbius, Klein and Poincare. ${ }^{7}$ This new conception of geometry was dominated by the idea of invariant form, namely of shapes and figures determined only up to some shared (algebraically expressed) features, e.g. the number of holes they contained. Klein's famous Erlangen program - which aimed to classify different geometries according to which symmetries the properties of its basic figures were invariant under - emerged as perhaps the most mature expression of this new kind of synthetic/algebraic geometry. And category theory - invented by S. Mac Lane and S. Eilenberg in the first half of the 20th century - then offered a framework in which to develop a vast generalization of Klein's ideas, thus giving rise, eventually, to the monumental re-imagining of algebraic geometry in the $20^{\text {th }}$ century by Grothendieck and his school. ${ }^{8}$ UF was born right here: both Makkai's and Voevodsky's foundational visions emerged directly out of the category-theoretic approach to algebraic geometry that was pioneered by Grothendieck and his school. In ways that have yet to be made precise, UF represents a return to a more Kantian view of mathematics, albeit one that also takes into account the revolutions in formal reasoning of the post-Hilbert era. But I will say no more about this

\footnotetext{
${ }^{6}$ The clearest exposition of Kant's views on the method of mathematics is to be found in the beginning of the Doctrine of Method and especially in [A713/B742]. There Kant explains that mathematics proceeds by constructing intuitions (e.g. specific triangles) adequate to a priori concepts, not by analyzing such concepts (which is the task of philosophy). (For a helpful overview see Carson [10].) Russell's very influential criticism of Kant was that a strong enough logic (e.g. first-order predicate logic) could compensate for this element of "construction" without invoking some kind of pure intuition provided by the human subject. This point of view came to be known as the "compensation thesis". For more discussion on Kant's views on geometry see the Parsons-Hintikka debate [17, 37] as well as Friedman's very influential recasting of the compensation thesis in $[13,14]$. It should also be made clear that the issue of whether or not Kant thought that demonstrations themselves also involved pure intuition (rather than just the construction of the concepts that they were to be applied to) remains a topic of controversy. For the latest installment, see Hogan [19].

${ }^{7}$ Strictly speaking, one must draw a distinction between topology (the study of shapes) and geometry (the study of distances) - in this very rough historical sketch I am including both under the banner of "geometry".

${ }^{8}$ Marquis [28] has made a compelling study of the connections between category theory, Kleinian geometry and Grothendieck's vision for algebraic geometry.
} 
here, leaving such explorations for future work.

\section{Outline of the Paper}

In Section 2 I will begin with a brief, non-technical introduction to Univalent Foundations. In Section 3 I will offer a pragmatist reading of the structuralist thesis in the philosophy of mathematics and then explain how it ought to apply to the foundations of mathematics. I will then articulate a precise criterion - (SFOM) - that a foundational system ought to satisfy if it is to be called structuralist in my sense. I will then examine the extent to which ZFC and ETCS fail (SFOM) (Section 3.1) and explain how (SFOM) shifts the focus from ontology to language, thereby transcending the debate about the foundational fitness of set theory vs. category theory (Section 3.2). In Section 4, I will argue that the axiom of univalence ensures that UF satisfies the "principle of isomorphism" which entails, roughly, that isomorphic objects are identical. I will spell out two further criteria that, if true, would ensure that UF comes closest to (SFOM) than other proposals. In Section 5 I argue that the second criterion is satisfied by describing a general method for formalizing criteria of identity as homotopy equivalences. Finally, in Section 6 , I will argue in favour of the first criterion by responding to several general challenges that have been put forward against the foundational aspirations of UF and by examining the scope of the general method of Section 5 .

\section{Univalent Foundations, HoTT and inten- sional MLTT}

A simple picture (sufficient for the purposes of this paper) of any foundation of mathematics (or foundational system) consists of the following elements:

Basic Language $\stackrel{\text {..used to express a... }}{\longrightarrow}$ Theory $\stackrel{\text {...that describes a... }}{\longrightarrow}$ Universe of Objects

(2)

where the "Basic Language" is a formal language of some sort, the "Theory" is a formal theory establishing a standard of rigour (usually through a notion of formal deduction) and the "Universe of Objects" is some intuitively comprehensible collection of objects such that all of currently practiced mathematics can be encoded in terms of them. Clearly, this is intended to be a very minimalist conception of a foundation of mathematics. One will usually want to impose extra conditions, e.g. that the "Basic Language" is finite or 
that the "Theory" is consistent or that the "Universe of Objects" is philosophically coherent in some appropriate sense. I will here refrain from doing so explicitly - this adds generality to the theses that I will argue for, but also, more importantly, indicates that the demand of (SFOM) that I will state in Section 3 below does not depend on any notion of a foundation that goes beyond this minimalist conception.

For set-theoretic foundations perhaps the most widely accepted set-up in terms of the above picture is the following

$$
\text { First-Order Logic with equality } \stackrel{\text {...used to express... }}{\longrightarrow} \mathrm{ZFC} \stackrel{\text {...that describes... }}{\longrightarrow} V
$$

where $V$ is the "real" cumulative hierarchy of sets. Of course, within settheoretic foundations both (1) and (2) are subject to variation. For example, (1) could be replaced by a Russellian type-theoretic syntax instead of firstorder logic (in which case (2) would, say, consist of asserting the axiom of reducibility among other axioms). Or we could maintain first-order logic as our (1) but use it to axiomatize different set theories in (2), e.g. ZF or intuitionistic ZF. ${ }^{9}$ Furthermore, one could argue philosophically about the metaphysics of (3), i.e. the "real nature" of $V$. What seemed inconceivable until very recently was a foundational system (in the above sense) in which component (3) was filled out by anything other than a universe of sets. To be sure, there was disagreement about what that universe of sets really should be. For example, should it understood as an ETCS-style category or a ZFCstyle cumulative hierarchy? But hardly anyone imagined the possibility of a foundation of mathematics in which the universe of basic objects was not meant to be understood as a collection/hierarchy/universe of sets of some kind.

With the Univalent Foundations of mathematics (UF), this becomes a real possibility. UF is a foundational proposal that differs from the standard set-theoretic set-up in all of the above components (1),(2) and (3). The standard set-up for UF - and the one I will consider for the purposes of this paper - is as follows

$$
\text { Intensional MLTT } \stackrel{\ldots \text { used to express... }}{\longrightarrow} \text { HoTT } \stackrel{\ldots \text {.that describes... }}{\longrightarrow} \infty \text {-Grpd }
$$

where $\infty$-Grpd is the "real" universe of homotopy types. Thus, by the term Univalent Foundations I will refer to a new foundation of mathematics

\footnotetext{
${ }^{9} \mathrm{I}$ am assuming here that the deductive system that we impose on the basic formal language comes in at stage (2) - but this is admittedly a difficult assumption to maintain in the case of MLTT where the rules of well-formedness of expressions coincide with the deductive rules. Still, even in dependent type theories used to formalize UF, there is a useful distinction to be made between "basic" type-formers (e.g. $\Pi, \Sigma$ or $W$-types) and type-formers or axioms added specifically with the homotopy interpretation in mind.
} 
of which HoTT is just one of many possible formalizations and intensional MLTT one of many possible basic languages. ${ }^{10} \mathrm{I}$ will now briefly introduce the above-stated components (1),(2) and (3) in the case of UF. ${ }^{11}$

"Intensional MLTT" refers to the cluster of formal systems that usually go under the banner of intensional Martin-Löf Type Theory (MLTT) [31]. MLTT was originally an attempt to give a constructivist ${ }^{12}$ and computerfriendly foundation of mathematics. It comes in extensional and intensional variants and we are here concerned exclusively with the latter. As a formal system, the basic objects of MLTT are types and terms. ${ }^{13}$ A judgment is a syntactic expression asserting a fact about terms and types in a certain context. The rules of the system tell us how to construct new types and terms from old ones using various type constructors, including but not limited to the empty type $(\mathbf{0})$, the singleton type $(\mathbf{1})$, product types, $\Pi$ - and $\Sigma$-types etc. There are two main innovations in MLTT that set it apart from the "type theories" that preceded it, e.g. Russell's and Church's. Firstly, MLTT introduces the notion of a dependent type. The idea is that we may "index" types by terms of another type - in which case we say that the family of types being indexed depends on the indexing type. As a formal judgement this would be written as $\Gamma, x: A \vdash B(x)$ Type which is to be read as follows: "In context $\Gamma$, given a term of type $x: A$ we can produce a type $B(x)$." Thus we say that the type $B$ depends on $A$. Secondly, MLTT introduces the notion of an identity type. As with other type constructors the identity type is governed by four deductive rules: formation, introduction, elimination and computation. The so-called formation rule, for example, says that given any type $A$ and any terms $a, b$ of type $A$, it is possible to form a type $a={ }_{A} b$, to be thought of as the type of "proofs of

\footnotetext{
${ }^{10}$ That said, I will often use the terms UF and HoTT interchangeably, i.e. I will often refer by the term UF to the particular formalization of UF as HoTT. This is in keeping with standard set-theoretic practice of referring to ZFC as "set theory".

${ }^{11} \mathrm{~A}$ full technical introduction to UF is neither possible nor necessary for the purposes of this paper. What follows is something slightly more structured than a glossary, meant to give some idea of what is meant by the term "Univalent Foundations" and to introduce some terminology peculiar to the formal apparatus it employs. For more detailed technical introductions see $[3,31,48,52]$.

${ }^{12}$ Martin-Löf's original name for his theory in [31] was Intuitionistic Type Theory - we shall not be concerned here with terminological issues concerning the difference between "constructivists" and "intuitionists". Suffice it to say that although MLTT has most often been associated with constructive/intuitionistic approaches to mathematics, UF is in no way limited to constructive/intuitionistic logic. Indeed, HoTT is perfectly consistent with an appropriately stated law of the excluded middle (cf. [48], Definition 3.4).

${ }^{13}$ One can think intuitively of types as sets and terms as elements of sets although in homotopy type theory this way of thinking is misleading since one can prove that there are types that are not $h$-sets (cf. [48], Example 3.1.9).
} 
identity" between $a$ and $b$.

By "Homotopy Type Theory" (HoTT) I will mean the formal system laid out in the Appendix of [48], namely intensional MLTT together with new deductive rules governing univalent universes and higher inductive types. ${ }^{14} \mathrm{~A}$ univalent universe is a universe of types (think: "class of sets") $\mathcal{U}$ satisfying the univalence axiom which states that the identity type between types is equivalent to homotopy equivalence. In HoTT the types of MLTT are no longer to be understood as sets, but rather as homotopy types or $\infty$-groupoids (for the purposes of this paper these two terms will be used interchangeably). This claim has been made precise in [21], where a model of HoTT is constructed in which types are interpreted as Kan complexes, which provide a widely accepted model for $\infty$-groupoids constructed settheoretically.

And what are homotopy types? Surely we cannot rely on their definition as Kan complexes inside ZFC. Nor should we rely on the mathematical definition of the homotopy group of a topological space, especially if that topological space is understood as a set of points with a topology. In other words: is there a "naive" way of understanding homotopy types? Broadly, yes: homotopy types are to be thought of as abstract shapes, just as sets are to be thought of as (abstract) collections. ${ }^{15}$ Why the qualifier "abstract"? Because homotopy types are - as the term itself suggests - meant to encode types of shapes that share some common structure. The homotopy type itself is the "skeleton" that is common to all particulars belonging to the given type - hence an abstract shape. But this does not mean that these "skeletons" are not amenable to concrete representations. In fact, the theory of $\infty$-groupoids emerged partly as an attempt to represent them as concrete combinatorial objects. What was scarcely imaginable was that these objects could then be employed as the basic objects of a foundations of mathematics, axiomatizable by a formal system in very much the same way as sets are. But it is this very possibility that allows us to consider defining structuralist foundations in the sense that I will now outline.

\footnotetext{
${ }^{14}$ It is important to note that this particular extension of MLTT is not the only homotopy type theory that formalizes UF. A recent alternative proposal is Cubical Type Theory $[6,11]$ designed with the specific intention of giving a constructive model of univalence. Nevertheless, for the purposes of this paper, nothing hinges on the choice of formalization and so we will identify the term HoTT with the formal system used in [48].

${ }^{15}$ For a more detailed exposition of a similar kind of view of homotopy types, as well as an argument for their fundamentality, see Marquis [30].
} 


\section{Structuralist Foundations of Mathematics}

Following Benacerraf [5], philosophers advocating a structuralist position in the philosophy of mathematics take themselves to be arguing in favour of some reasonable variation of what we may call the structuralist thesis: Mathematical properties of mathematical objects are concerned with the relations these objects bear to each other, rather than what these objects are. Parsons [36], for example, expresses this as "the view that reference to mathematical objects is always in the context of some background structure, and that the objects involved have no more to them than can be expressed in terms of the basic relations of the structure" and Resnik [40] as the view that "the objects of mathematics [...] are structureless points or positions in structures [that] have no identity or features outside of a structure."

Rather ironically, several disputed structuralist positions are then carved out through ontological disagreements on what "structure" itself is. There is, for instance, disagreement over whether structures are possibilia (Hellman [16] and, more obliquely, Putnam [38]) or Platonic natureless abstractions (Shapiro's ante rem structuralism in [43]) or even just sets (following in the Bourbakian tradition). This direction takes the structuralist thesis to be an observation about ontology: mathematical objects are structures. Put differently: what it is for something to be a structural property is to be determined by figuring out what structure means.

On the other hand we may take the structuralist thesis to be an observation about mathematical practice. Namely: the only mathematical properties of a mathematical object that are meaningful in practice are those that are invariant under "isomorphism", where "isomorphism" is a place-holder for the relevant criterion of identity for the type of object under consideration and where what is "relevant" is to be understood by recourse to mathematical practice. For example, the only meaningful mathematical properties of a group are those that are invariant under group isomorphism and the only meaningful mathematical properties of dense linear orders are those that are invariant under order-preserving bijections. Group isomorphism and orderbijection are relevant criteria of identity because practicing group theorists or number theorists (working, say, over $\mathbb{Q}$ ) would agree that they preserve the truth of the statements they are interested in. In short, we may define as structural those properties that mathematicians themselves would find meaningful (or "sensible"). Let me call this the practical reading of the structuralist thesis.

There are two ways to make good on the practical reading of the structuralist thesis. Firstly, informally. This is roughly the direction taken by Awodey [2] and McLarty [32,33] ("schematic" or "categorical" structuralism) 
and more recently by Burgess $[8,9]$ ("permanent parameter" structuralism). Secondly, formally - this is where my interests lie. The focus here is more on the foundations of mathematics rather than on the philosophy of mathematics. Specifically, we take the practical reading of the structuralist thesis to indicate a "design constraint" for a foundation of mathematics. The motivating problem is thus to create a foundational system (in the sense of Section 2) such that any grammatically well-formed property about a mathematical object is invariant under the appropriate criterion of identity for that object (as those are formalized in the given system).

With this in mind I define a structuralist foundation of mathematics to be a foundational system $\mathcal{S}$ that satisfies the following property:

(SFOM) Any theoretical context can be naturally formalized in $\mathcal{S}$ in such a way that any grammatical property of an object in $\mathcal{S}$ is invariant under the relevant criterion of identity in that context.

I will now clarify some of this terminology. A "foundational system" refers to some formal system sufficiently expressive to encode all of (currentlypracticed) mathematics, i.e. component (2) of a foundation of mathematics as outlined in Section 2. "Object" refers to a mathematical object (e.g. a group, a number, a manifold) and a "grammatical property" refers to a correctly formed expression in $\mathcal{S}$ (e.g. a well-formed formula $\phi$ in $\mathrm{FOL}_{=}$). By "theoretical context" I mean a particular mathematical (sub-)discipline (e.g. group theory or topology). By "criterion of identity" I mean the notions of "sameness" employed by practitioners of those mathematical disciplines (e.g. group isomorphism or homeomorphism of spaces). ${ }^{16}$ Finally, "invariance" of a grammatical property $P$ means that if $P$ holds of $x$ in $\mathcal{S}$ and if $x \cong y$ in $\mathcal{S}$ then $P$ also holds of $y$ (where $x, y$ and $\cong$ are the formalizations in $\mathcal{S}$ of two objects in, and the criterion of identity of, a given theoretical context).

It is important to be clear that (SFOM) asserts the existence of invariant formalizations, i.e. those that satisfy the invariance property outlined in its statement. (SFOM) does not assert that all possible formalizations are invariant. As the last sentence makes clear, (SFOM) is a principle relating mathematical practice to the formalization of mathematical practice in $\mathcal{S}$.

\footnotetext{
${ }^{16}$ It is an interesting question whether a choice of context determines the criterion of identity or vice versa, but I will not get into this here. Furthermore, it is clearly an oversimplification to say that mathematical disciplines are rigidly demarcated by a specific type of structure they study up to a specific criterion of identity. For instance, how would one demarcate number theory in those terms? Or algebraic geometry? Nevertheless, nothing of substance hinges on the accuracy of my sociological analysis - its purpose is merely to clarify the concepts of "theoretical context" and "criterion of identity" as they will be used below.
} 
In order to make sense of (SFOM) these two concepts - practice vs. formalization of practice - must be kept apart. For example, the "natural numbers" as understood by the practicing number-theorist are not to be identified with a model of Peano Arithmetic studied by the set theorist. Thus, the "invariance" that (SFOM) demands is a statement about objects and criteria of identity as formalized by $\mathcal{S}$. The dominance of set theory in the last halfcentury has often tempted philosophers to identify mathematical concepts with their set-theoretic formalizations - it is of paramount importance that we resist this temptation here.

Furthermore, we must note that the mere existence of invariant formalizations is too weak a demand to prove interesting. After all, almost any foundational system can reasonably claim the mere existence of invariant formalizations, since anything can be called a formalization, however contrived. For example, we could say that Peano Arithmetic provides invariant formalizations for mathematical contexts simply by coding mathematical statements as (lists of) numbers in a particular way. To avoid such cases, (SFOM) imposes the additional constraint of requiring the existence of "natural" formalizations. This constraint demands that the required invariant formalizations are:

1. Uniform: As much as possible, the invariant formalizations are obtained through some general method of encoding that applies equally well to all mathematical structures of interest

2. Native: The practicing mathematician would find the formalized notions obtained through these invariant formalizations easily comprehensible

One may summarize the requirement of naturality as follows: (SFOM) demands both the natural existence of invariant formalizations (uniformity) and the existence of natural invariant formalizations (nativity).

Finally, let me note that as a criterion for foundational systems, (SFOM) clearly depends on the current practice of mathematics. For it is mathematical practice that determines not only which notions have to be formalized but also which formalizations are native and whether or not they are invariant. As such, the ambition of (SFOM) cannot exceed our grasp of current mathematical practice. Mathematical practice evolves and so do the structures that mathematicians find useful to work with. So the most reasonable way to understand (SFOM) is as the demand that for most of the mathematical structures of current interest there exists a uniform method for producing native invariant formalizations. To speak of a foundation that can do this for all mathematical structures of past, present and future interest is a goal 
worthy only of an incredulous stare - at least insofar as one remains grounded in the practice of mathematics, as we intend to do.

Indeed, (SFOM) is put forward as an ideal to aspire to rather than a realistic goal. In that sense, it is similar to the ideal of more traditional foundations, summarized nicely in [34] as the attempt to find "consistent, finitely axiomatizable theories [into which as many theories can be interpreted]." Full assurance of consistency is never attainable by Gödel's theorems, but considerations of simplicity, coherence etc. may provide better or worse reasons to believe in it. Analogously, it is hard to imagine that (SFOM) will ever be decisively satisfied, i.e. for any theoretical context. Rather, we will seek foundational systems into which there are natural invariant formalizations of ever more theoretical contexts. What I will establish in this paper is that UF achieves just that, i.e. it comes closest to (SFOM) than all other foundational proposals even if it remains shy of the ideal. Nevertheless, UF represents a watershed moment since it appears that if one is to make any progress with respect to $(\mathrm{SFOM})$ one has to move beyond a broadly Cantorian picture of the foundations of mathematics. To see this, we will now examine the extent to which current set-theoretic foundations fail (SFOM).

\subsection{ZFC and ETCS are not structuralist foundations}

It is relatively straightforward to see how ZFC fails (SFOM) ${ }^{17}$ Let our "theoretical context" be traditional group theory, i.e. the study of groups up to isomorphism exemplified by the kind of methods and questions that, say, go into the monumental proof of the classification of finite simple groups. In this theoretical context the "objects" of study are groups and the "criterion of identity" is group isomorphism. In ZFC, a group is formalized by first-order formulas defining what it is for a set to have group structure. More precisely, we define a three-place predicate

$$
\operatorname{Group}(x, *, 0) \equiv * \in(x \times x \rightarrow x) \wedge 0 \in x \wedge \operatorname{GroupAxioms}(*, 0)
$$

with the obvious abbreviations. What it is to be a group in ZFC is to satisfy this predicate. Similarly, what it is for two groups to be isomorphic is formalized by a predicate

$$
\operatorname{Iso}(x, y) \equiv \operatorname{Group}(x) \wedge \operatorname{Group}(y) \wedge \exists f \in(x \rightarrow y)(\operatorname{homom}(f) \wedge \operatorname{bijective}(f))
$$

\footnotetext{
${ }^{17}$ Everything I say applies to any standard set-theoretic foundation. I pick ZFC for this example merely for its "brand recognition".
} 
again with the obvious abbreviations and suppressing explicit mention of the group operations. ${ }^{18}$ What it is for two sets $x, y$ to be isomorphic as groups in ZFC is to satisfy $\operatorname{Iso}(x, y)$. Furthermore, by a "grammatical property of an object" in ZFC we mean simply a one-place open formula $\phi(x)$ in the language of ZFC and for such a grammatical property $\phi(x)$ to be "invariant under the relevant criterion of identity" we mean that

$$
\mathrm{ZFC} \models(\operatorname{Iso}(x, y) \wedge \phi(x)) \rightarrow \phi(y)
$$

So with all this in mind now take

$$
\phi(x) \equiv 1 \in x
$$

to be such a "grammatical property" and consider $\mathbb{Z}$ and $2 \mathbb{Z}$ with their canonical (additive) group structure. ${ }^{19}$ We have

$$
\mathrm{ZFC} \models \mathrm{Iso}(\mathbb{Z}, 2 \mathbb{Z})
$$

But we also have

$$
\mathrm{ZFC} \models \phi(\mathbb{Z})
$$

whereas

$$
\mathrm{ZFC} \not=\phi(2 \mathbb{Z})
$$

In ZFC therefore we have that $\phi(\mathbb{Z})$ is true whereas $\phi(2 \mathbb{Z})$ is not true even though $\mathbb{Z} \cong 2 \mathbb{Z}$, i.e. even though $\mathbb{Z}$ and $2 \mathbb{Z}$ are identical with respect to the relevant group-theoretic criterion of identity. In short, grammatical properties in ZFC are not invariant with respect to group isomorphism as it is formalized in ZFC. Therefore, ZFC fails (SFOM). ${ }^{20}$

The above analysis carries over almost verbatim to so-called "structural set theories" [23,33] of which Lawvere's ETCS [22] is perhaps the paradigm. To see this, let $o$ stand for the canonical arrow

$$
o: 1 \rightarrow \mathbb{Z}
$$

\footnotetext{
${ }^{18} \mathrm{I}$ am being a bit quick here, for the sake of exposition. Strictly speaking, the actual operation and identity elements of groups $x, y$ would have to be used in defining what it is for a function $f$ to be a homomorphism.

${ }^{19} \mathrm{By}$ " 1 " here I mean the set corresponding to the successor of zero in some choice of a model of arithemtic inside ZFC, e.g. the singleton set $\{\varnothing\}$. By $\mathbb{Z}$ and $2 \mathbb{Z}$ I mean the formalizations of the additive groups of integers and even integers respectively.

${ }^{20}$ Of course, one could argue that there are non-standard ways of formalizing groups and group isomorphisms in ZFC such that the required invariance holds. However, it is highly doubtful that such methods would be natural (i.e. uniform and native) in the sense demanded by (SFOM).
} 
and let

$$
\phi(x) \equiv \operatorname{cod}(o)=x
$$

where $\operatorname{cod}$ is the codomain operation of arity Arrows $\rightarrow$ Objects that takes an arrow to its codomain. ${ }^{21}$ Then it is immediately seen that

$$
\operatorname{ETCS} \models \phi(\mathbb{Z})
$$

whereas

$$
\text { ETCS } \forall \bullet \phi(2 \mathbb{Z})
$$

Thus, just like ZFC, ETCS also fails (SFOM).

That it does so illustrates an important omission on the part of those who have advocated ETCS (and its variants) as "structural" set theories. A commonly-employed argument in making such a claim is that in ETCS the natural numbers $\mathbb{N}$ are only defined up to isomorphism. More precisely, in ETCS one defines the natural numbers by defining a natural numbers object (NNO) via a universal property. Thus, one of the axioms of ETCS asserts that such an NNO satisfying the requisite universal property exists. It is indeed the case that this axiom determines an NNO only up to isomorphism - but then again so does the axiom of infinity in ZFC "determine" $\aleph_{0}$ up to bijection. And just as the axiom of infinity in ZFC does not prevent us from expressing properties that are true of one countably infinite set but not of another, so does the axiom for the NNO not prevent us from expressing properties that are true of one NNO but not of another (necessarily) isomorphic to it. Similarly, the axiom of an NNO no more prevents us from stating properties that separate $\mathbb{Z}$ from $2 \mathbb{Z}$ than the axiom of infinity prevents us from stating properties separating $\{\varnothing,\{\varnothing\},\{\{\varnothing\}\}, \ldots\}$ from $\{\varnothing,\{\varnothing\},\{\varnothing,\{\varnothing\}\}, \ldots\}$. This indicates that the real culprit is not the notion of elementhood of extensional set theories like ZFC but rather the availability of a global (untyped) identity predicate. In the case of ZFC this identity is intended to apply to individual sets and in the case of ETCS it is intended to apply to individual functions between sets, but this makes little difference with respect to (SFOM).

One might object that this criticism of ZFC and ETCS relies essentially on their being formulated in first-order logic. This is correct: the criticism I have presented so far applies to languages with a global (untyped) identity predicate and not just to certain theories that can be formulated in terms of such languages. There are alternative ways of formulating some of these theories that make no essential use of first-order logic. For example, ETCS

\footnotetext{
${ }^{21}$ I am glossing over the details of the exact presentation of ETCS as a many-sorted first-order theory. For a clear and concise presentation see Palmgren [35].
} 
can be formulated on top of a simple dependent type theory or, alternatively, as a theory in Makkai's First-Order Logic with Dependent Sort (FOLDS).

Yet although such formulations do avoid the kind of objection outlined above for set-level structures (such as $\mathbb{Z}$ ) they still do not avoid it for higherlevel structures like categories. The reason, essentially, is once again that such formulations contain something that behaves very much like an untyped identity predicate. To illustrate, consider the axiomatization of ETCS as the FOLDS $\mathcal{L}_{\text {abset }}$-theory $\mathbb{T}_{\text {abset }}$ of abstract sets in [27]. One can show that any $\mathcal{L}_{\text {abset-sentence is invariant under isomorphism of diagrams in any model }}$ $\mathcal{E}$ of $\mathbb{T}_{\text {abset }}$. Thus, any set-level structure that can be expressed in terms of an internal diagram in $\mathcal{E}$ will satisfy the required invariance property. Formalizing such set-level structures in terms of diagrams in $\mathcal{E}$ thus gives us a (SFOM)-compatible general method for formalizing all set-level structures. However, this invariance does not extend beyond isomorphism of diagrams, and therefore becomes problematic when we want to axiomatize higher-level structures like categories.

To see this, recall that a category internal to another category with appropriate structure is given by a diagram

$$
\ldots \longrightarrow C_{1} \times C_{0} C_{1} \stackrel{m}{\longrightarrow} C_{1} \underset{\underbrace{\stackrel{d}{\rightleftarrows}}_{c}}{\stackrel{d}{\rightleftarrows}} C_{0}
$$

satisfying certain conditions (where $d$ is for "domain", $c$ is for "codomain", $i$ is for "identity" and $m$ is the composition operation). As explained above, FOLDS $\mathcal{L}_{\text {abset-sentences will be invariant only under isomorphism of such }}$ diagrams. This means that the desired invariance will hold only if the sets of objects of two such internal categories are isomorphic. Thus, $\mathcal{L}_{\text {abset }}$-formulas will be invariant only up to isomorphism, rather than equivalence, of categories and it is the latter that is usually the criterion of identity one wants to consider in this theoretical context. ${ }^{22}$ So in particular in $\mathbb{T}_{\text {abset }}$ it is possible to define the category $\mathbf{1}$ consisting of one object and one identity morphism and the category $\mathbf{1}_{i}$ with two objects and an isomorphism between them, and

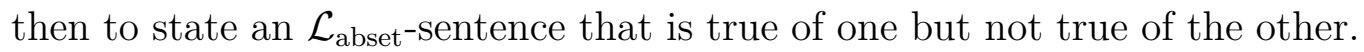
The same reasoning can be repeated for similar such "type-theoretic" axiomatizations of ETCS, thus casting a doubt on their adherence to (SFOM) beyond set-level structures.

One point that may be granted, however, is that structural set theories like ETCS certainly do better than ZFC in this respect since it is not even possible to formulate the latter in this "half-way" manner. For even if ZFC is

\footnotetext{
${ }^{22}$ As we shall see in Section 5 , the situation is slightly more subtle.
} 
formulated on top of a type theory, the culpable distinctions can still be made from the very beginning because we will have available to us the membership relation " $\in$ ". Nevertheless, neither approach, whether fully first-order or "half-way", manages to avoid making irrelevant distinctions at some point. If we are to make any progress, it appears that we need to entirely reconsider the basic language on which these foundations are based, rather than this or that axiom we can express using it.

\subsection{From Ontology to Language}

Clearly, then, (SFOM) is not at all intended to separate set-theoretic foundations from category-theoretic foundations of mathematics. As such, the demand for (SFOM) transcends the debate between category-theoretic and set-theoretic foundations of mathematics. But have we gone too far then? By lumping category-theoretic and set-theoretic foundations together, what kind of circle have I drawn around them? Is there anything left on the other side?

In a way, (SFOM) urges a "linguistic turn" in the foundations of mathematics. Forget about the metaphysical view that a certain foundation takes of its basic objects. Instead, focus on what properties of those objects its language allows it to express. To be sure, there is a significant metaphysical difference in how a material set theory and a structural set theory view the objects they are trying to axiomatize. But it is their linguistic promiscuity that (SFOM) rejects, not their intended metaphysics. Structuralism in the sense of (SFOM) is thus best thought of as a constraint on language: we want to stop ourselves from being able to express non-structural properties of objects.

One may now wonder: if this constraint on language is all that is required, then why not just replace objects with isomorphism classes? Both set-theoretic and category-theoretic foundations allow us to do this and doing so certainly would satisfy the criterion that (SFOM) demands of us: for if we formally identify two things, then surely we won't be able to say something of one that is not true of the other since there will not even be an "other" of which to say anything at all.

The problem is that treating isomorphic objects as actually identical involves essential loss of information. To see this, we must first note that there are two ways of treating isomorphic objects as identical. Firstly, by simply lumping them together in isomorphism classes and ignoring non-identity isomorphisms between them. For example, if I were to replace $\mathbb{Z}$ with its isomorphism class as a group, I am no longer able to ask how many homomorphisms there are, say, between $\mathbb{Z}$ and $2 \mathbb{Z}$ - indeed I cannot really talk 
about $\operatorname{Hom}(\mathbb{Z}, 2 \mathbb{Z})$ at all. However, that $\mathbb{Z}$ and $2 \mathbb{Z}$ are isomorphic as groups is a non-trivial mathematical fact - not being able to express it at all is clearly a crippling limitation.

Secondly, and less naively, we can treat isomorphic objects as identical by identifying isomorphic objects without forgetting about non-identity isomorphisms between them. This is essentially what happens when one moves from a category $\mathcal{C}$ to a skeleton $s k(\mathcal{C})$ : isomorphisms between objects $a, b$ in $\mathcal{C}$ become (non-identity) automorphisms in $s k(\mathcal{C})$. Thus, although we have "fewer" objects than we started with we have just as many arrows and this ensures that no essential information has been lost. Unfortunately, the problem reappears when we then want to talk about further structure on $s k(\mathcal{C})$. For example, even if we move from the category of sets Set to one of its skeletons $\mathcal{S}$ (thus identifying bijective sets) this does not ensure that isomorphic groups defined on $\mathcal{S}$ are now also equal. To achieve this, we would have to then consider a skeleton of the category $\operatorname{Grp}(\mathcal{S})$, and so on. ${ }^{23}$ This brings into sharp relief the following crucial distinction: identifying isomorphic objects is not at all the same thing as making isomorphic objects indistinguishable. The former involves loss of information, whereas the latter does not. Doing the former is possible even in ZFC or ETCS but doing the latter is not.

In other words, (SFOM) does not express an appeal to identify isomorphic objects. Quite the contrary: it is of paramount importance, mathematically, to be able to state an isomorphism between two mathematical objects, e.g. between $\mathbb{Z}$ and $2 \mathbb{Z}$. And in order to even be able to state such an isomorphism, clearly we do need to view $\mathbb{Z}$ and $2 \mathbb{Z}$ as distinct on some level. The reader has perhaps already felt some unease about this point. We are speaking of making isomorphic objects indistinguishable, but if an isomorphism between them is to be construed as a substantive statement, then surely we must distinguish them somehow. And if we can distinguish them somehow, even if they are isomorphic, then what kind of structuralists are we? Even worse, if we buy into Quine's [39] slogan that there should be no entity without identity, then to accept that our isomorphism relates entities is to pre-suppose that there is some notion of identity (other than isomorphism) that allows us to view them as distinct to begin with. We appear to have fallen into a trap.

At this point I want to say that we have been trapped only by an excessive pre-occupation with ontology. After all, what (SFOM) demands is quite simple: as long as we can establish an isomorphism between two objects, then there is no grammatical property of one that does not also hold of the other. This says nothing about what these objects are. That is not

\footnotetext{
${ }^{23} \mathrm{I}$ am indebted to an anonymous referee for raising this point.
} 
to say that we don't know what these objects are: they are formalized versions of constructions employed by mathematicians. "And what are these constructions that mathematicians employ?" Enough. It is irrelevant to our purposes. All we require is that whatever these constructions are (Platonic abstractions, mental occurences, intersubjectively valid concepts etc.) the people that use them - the mathematicians - agree that they have been adequately formalized in the given foundational system we are studying. ${ }^{24}$ Is it possible to speak of such objects without commiting ourselves to a notion of identity that is finer than any isomorphism that we could establish between them a posteriori? I think it is, at least in the following uncontroversial sense: whatever notion of identity we require in order to distinguish between two presentations of isomorphic objects, it is not necessarily a notion of identity relevant in the mathematical practice from which the particular notion of isomorphism emerges. For example, we have a way of presenting $\mathbb{Z}$ as a group and we also have a way of presenting $2 \mathbb{Z}$ as a group. But when we are doing group theory these distinct ways of presenting these isomorphic groups should not automatically commit us to regarding propositions like " $\mathbb{Z} \neq 2 \mathbb{Z}$ " as relevant to group theory. ${ }^{25}$

Therefore, in order to avoid the Quinean trap, we should make this most minimal of pre-suppositions: that there are distinct ways of presenting objects without committing ourselves to the existence of a significant proposition that distinguishes them. This presupposition allows us to say: if we can establish an isomorphism between these two distinct ways of presenting this object, then we should not be able to state anything in our language that is true of one presentation but not true of the other. And this is exactly what (SFOM) demands. Even the word "object" in the previous sentence is something of a distraction. We can drop talk of objects altogether. Forget about referents/extensions. All we have are senses/intensions - and what (SFOM) wants is that if two such senses/intensions can be shown to be "isomorphic" then the required invariance property holds. In shifting our focus from ontology to language, we can thus completely invert the Quinean motto: there

\footnotetext{
${ }^{24}$ This attitude towards mathematical objects is inspired by Burgess' permament parameter structuralism as elaborated in [9]. What I am saying, roughly, is that mathematical objects should be understood as parameters, about whose nature we do not care as long as they behave the way we expect them to. And this allows us to speak of "alternative presentations" of such objects without committing ourselves to a criterion of identity between them.

${ }^{25}$ Of course, in the context of number theory a proposition like " $\mathbb{Z} \neq 2 \mathbb{Z}$ " is highly relevant indeed since we care very much to distinguish odd from even integers, e.g. when we state Goldbach's conjecture. But in the context of number theory group isomorphism is not, in general, the relevant criterion of identity.
} 
is no identity without entities. ${ }^{26}$ In other words, if we think of "identity" between two objects $A$ and $B$ as no more than what allows us to transfer any properties of $A$ to $B$ and vice versa, then what (SFOM) demands is that "identity" become identified with "isomorphism". Or, more accurately: that "identity" become isomorphic with "isomorphism". As I will now go on to argue, this is exactly what the axiom of univalence in UF achieves.

\section{$4 \quad$ Univalence and (SFOM)}

What does the axiom of univalence say? It says, quite literally, that to ask of two types whether or not they are identical is the same as asking whether or not they are "isomorphic." In symbols this can be expressed as follows. For any types $A$ and $B$ (understood as terms in a universe of types $\mathcal{U}$ ) the following holds:

$$
(A=\mathcal{U} B) \cong(A \cong B)
$$

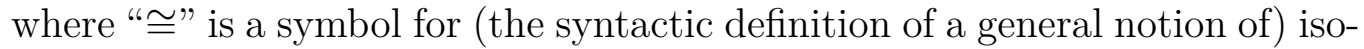
morphism and " $=\mathcal{U}$ " is the symbol for the identity type in MLTT formed in $\mathcal{U}$ (when $A$ and $B$ are regarded as its terms). Thus, when properly paraphrased, the axiom of univalence (UA) asserts the following: Identity is isomorphic to isomorphism. ${ }^{27}$

The statement of univalence might immediately appear baffling. For it seems as though UA is asserting an isomorphism between two things, one of which is not at all clearly a structure. For suppose one accepts that the type " $A \cong B$ " of isomorphisms is a structure of some sort. Then what about the identity $A=\mathcal{U} B$ ? Identity, surely, is a proposition of some sort. It is either true or false. How can a proposition be isomorphic to a structure? It appears that the statement of univalence involves a category mistake.

Strange as it may initially appear, the answer is that in UF (and in intensional MLTT more generally) identity is not (generally) a proposition - it just $i s$ a structure. Depending on $A$ and $B, A=\mathcal{U} B$ could turn out to be a structure containing a lot more information than merely whether it is inhabited ("true") or not ("false"). For example, there could be distinct terms of type $A=\mathcal{U} B$, which one can think of as distinct proofs of the identity between $A$ and $B$. In short, for the statement of UA to make sense

\footnotetext{
${ }^{26}$ More accurately, but less sonorously: there is no criterion of identity without names for entities.

${ }^{27}$ As has already been noted, the precise statement of univalence asserts that identity is isomorphic to isomorphism by asserting that a canonical map from identities to isomorphisms is an isomorphism. In other words, univalence asserts that a particular (canonical) map is an isomorphism, and not merely the existence of an isomorphism.
} 
one must accept the point of view of identity-as-structure. And after doing so, it is not too much of a stretch to view UA as analogous to the axiom of extensionality in set theory. Just as the latter provides a definition of (the previously underdetermined) equality of sets ("Two sets are equal if and only if they have the same elements") so does UA provide a definition of the (previously underdetermined) identity of types in intensional MLTT. The key difference, however, is that the "definition" that UA provides does not assert the logical equivalence of one fact ("being equal sets") to another fact ("having the same elements"). Instead, it asserts the isomorphism of one structure ("identity type") to another structure ("type of isomorphisms").

So now let us ask: what can univalence do for us when it comes to (SFOM)? Firstly, UA ensures that any grammatical property $P$ expressible in HoTT that is true of a type $A$ is also true of a type $B$ that is isomorphic to $A$. Following Awodey [3], we may call this fact the Principle of Isomorphism (PI).$^{28}$ As shown by Awodey [3] in quite some detail, UF satisfies (PI). How? Roughly, because a proof of isomorphism between $A$ and $B$ can give rise, through UA, to a proof of identity between $A$ and $B$. And since properties are invariant under identity we get that if $P$ holds of $A$ then it also holds of $B$. Thus, any grammatically well-formed property in HoTT is invariant under isomorphism.

Furthermore, we must now note that the term "isomorphism" in the statement of (PI) and in how we have been using it above actually refers to a much more general notion. This more general notion is homotopy equivalence which can be understood as the "correct" criterion of identity for homotopy types. The notion of homotopy equivalence originates in algebraic topology but it can be expressed syntactically in intensional MLTT without the addition of extra non-logical symbols. That this can be done is one of the key insights that led to the homotopy interpretation of MLTT. Thus, given any two types $A, B$ in $\mathcal{U}$ it is possible to define a type of homotopy equivalences between $A$ and $B$ using only the symbols of MLTT. One can certainly continue to think of it as the type of "isomorphisms" between $A$ and $B$ as long as it is clear that it is a more general notion than what is usually understood by the term "isomorphism", i.e. structure-preserving bijections between sets. But - as Awodey himself notes - homotopy equivalence does specialize to more familiar notions in more familiar cases. For example, bijections between sets can be seen as special instances of homotopy equivalence and so can logical equivalence of propositions. ${ }^{29}$

\footnotetext{
${ }^{28} \mathrm{In}[3]$, Awodey refers to (PI) as the Principle of Invariance but this is merely a terminological difference.

${ }^{29}$ In keeping with the notation above, I will continue to use $A \cong B$ to denote the type of homotopy equivalences, except when ambiguity might arise, as in Section 5, where I will
} 
An important issue here is that homotopy equivalence, as defined in HoTT, only meaningfully applies to pairs of types $A, B$ in a (univalent) universe $\mathcal{U}$ and not to terms of such types. Therefore, it makes prima facie no sense to speak of homotopy equivalence between two terms $a, b$ of a type $A$ in $\mathcal{U}$. We shall however stipulate here that we will regard such instances as degenerate cases of the notion of homotopy equivalence. In other words, we will allow ourselves to refer to the identity type $a={ }_{A} b$ for terms $a, b: A$ as the type of homotopy equivalences between them; from now on the term "homotopy equivalence" will be understood in this expanded sense and we will use the notation $a \cong b$ to denote it. One can of course take this as no more than a terminological convention. But since the way in which I will argue for (CI) below depends on it I ought to say a few words about why it is a reasonable point of view to adopt. The reason, quite simply, is that types themselves can be regarded as terms of a (univalent) universe $\mathcal{U}$ and in that case homotopy equivalence does apply to terms of a type. From a topological point of view, anything can be regarded as a point or as a space; viewing spaces as points in some larger space allows us to talk about homotopy equivalence between points. ${ }^{30}$

With this in mind, one cannot fail to notice the similarity between (PI) and (SFOM) when the latter is specialized to UF. For what we have is

(PI) Any grammatical property of an object in HoTT is invariant under homotopy equivalence

and what we need is

\section{(SFOM-UF) Any theoretical context can be naturally for- malized in HoTT in such a way that any grammatical prop- erty of an object in HoTT is invariant under the relevant cri- terion of identity in that context}

In order to argue that UF is a structuralist foundation in my sense it suffices to show that $(\mathrm{PI}) \Rightarrow(\mathrm{SFOM}-\mathrm{UF})$ and as the above comparison makes clear, to do so one needs to argue that

(F) any theoretical context can be naturally formalized in HoTT

and

use the more standard " $\simeq$ ".

${ }^{30}$ This can even be formalized in point-set topology for sufficiently nice spaces, by regarding their points as singleton subspaces and showing that equal such points are (trivially) homotopy-equivalent as singleton subspaces. 
(CI) in such a way that the relevant criterion of identity for that context coincides with homotopy equivalence.

I will investigate the extent to which $(\mathrm{F})$ is true in Section 6 . The following Section is devoted to showing that (CI) is true.

\section{Criteria of Identity as Homotopy Equiva- lence}

In order to establish (CI) we must provide a general method for formalizing mathematical structures such that their formalized criterion of identity coincides with homotopy equivalence. In what sense of "coincide"? At the very least, in the sense of "being homotopy equivalent to" it.

The set-up is as follows: we are given informally a class $O_{\text {inf }}$ of objects and a specification of a criterion of identity $\sim_{\text {inf }}$ for these objects. ${ }^{31} \mathrm{~A}$ formalization of the theoretical context given by $O_{\text {inf }}$ and $\sim_{\text {inf }}$ then consists of a type $O_{\mathrm{UF}}$ in $\mathcal{U}$ together with a relation $\sim_{\mathrm{UF}}$ between any two terms of that type. Formally, we can write this relation as

$$
\sim_{\mathrm{UF}}: \mathrm{O}_{\mathrm{UF}} \rightarrow \mathrm{O}_{\mathrm{UF}} \rightarrow \mathcal{U}
$$

and it is important to note that this relation is not necessarily a proposition. Namely, for any $a, b: O_{\mathrm{UF}}$ the most we can tell about $a \sim_{\mathrm{UF}} b$ is that it is a type in $\mathcal{U} .^{32}$

What remains now is to show that $\sim_{\text {UF }}$ coincides with homotopy equivalence. Clearly there are many ways to make this precise, as we have not restricted ourselves to a single way of understanding what it means for $\sim_{\mathrm{UF}}$ to "coincide with homotopy equivalence." I will make it precise in the following way:

\footnotetext{
${ }^{31}$ What this "class" is and how exactly it is "specified" we leave open. At the very least, there should be some consensus among practicing mathematicians that studying objects of type $O_{\text {inf }}$ under $\sim_{\text {inf }}$ constitutes a legitimate specialization. For instance, studying groups under group isomorphisms ("group theory") or studying topological spaces up to homotopy equivalence ("homotopy theory").

${ }^{32}$ Just like what was said about identity types in the beginning of Section 4, a criterion of identity is also to be understood as a structure rather than a proposition. Of course, if $\sim_{\text {UF }}$ is to be meaningfully regarded as a criterion of identity then we will usually assume that it is at least reflexive, symmetric and transitive (in the type-theoretic sense). But we also allow for criteria of identity that satisfy much stronger properties, e.g. identity systems in the sense of [48], Definition 5.8.3.
} 
i. Define $\sim_{\mathrm{UF}}$ as a type $\cong_{O}$ canonically definable from $O_{\mathrm{UF}}$ and canonically equivalent to the type of homotopy equivalences on $O_{\mathrm{UF}}{ }^{33}$

ii. Provide a general method that exhibits $\cong_{O}$ as the correct formalization of $\sim_{\text {inf }}$ under a certain way of understanding $O_{\text {inf }}$ and $\sim_{\text {inf }}$.

The first task is taken up in Section 5.1 and the second task in Section 5.2.

\subsection{Component-wise isomorphism as a criterion of iden- tity}

To define $\cong_{O}$ we must consider two cases. Firstly, that in which $O_{\text {inf }}$ is formalized as a type that does not involve collecting other types together. For example, if $O_{\text {inf }}$ is the class of natural numbers $\mathbb{N}$ and $\sim_{\text {inf }}$ is the relation of equality of numbers - in which case the "theoretical context" is, say, number theory. Secondly, that in which $O_{\text {inf }}$ is formalized as a collection of types with certain additional structure, satisfying certain properties. For example, if $O_{\text {inf }}$ is the class of groups and $\sim_{\text {inf }}$ is the relation of group isomorphism - in which case the "theoretical context" is, say, group theory. In model-theoretic terms we can think of types of the first kind as theories with an intended model (e.g. arithmetic) and types of the second kind as theories without an intended model (e.g. group theory).

In the first case, $O_{\mathrm{UF}}$ will be some type constructed in UF without using a $\Sigma$-constructor over $\mathcal{U}$. In other words, given terms $a, b: O_{\mathrm{UF}}$ their identity type $a={ }_{O_{\mathrm{UF}}} b$ is not equivalent to some type that itself contains an identity type between two types in $\mathcal{U}$. To illustrate: if $O_{\mathrm{UF}} \equiv \mathbb{N}$ then the identity type between any two terms $n, m: \mathbb{N}$ is simply the identity type formed in the usual way. It is not equivalent to some other type that is constructed out of other identity types. Thus, as long as we are formalizing a theoretical context of the first kind, the relevant criterion of identity will be given by the identity type for terms of that type, just as in intensional MLTT. Since we have stipulated that in such cases we will consider the identity type as a degenerate version of homotopy equivalence, we get a formalization of the kind demanded by (CI).

In the second case, we are studying a class of structures best formalized as a collection of types. Here $O_{\mathrm{UF}}$ will be of this general form:

$$
S \equiv \sum_{A: \mathcal{U}} \operatorname{Struc}(A) \times \operatorname{Prop}(A)
$$

\footnotetext{
${ }^{33}$ Where "homotopy equivalences" are understood in the expanded sense outlined in Section 4.
} 
i.e. a collection of types $A$ in a universe $\mathcal{U}$ that have a certain structure $\operatorname{Struc}(A)$ satisfying certain properties $\operatorname{Prop}(A) \cdot{ }^{34}$ How can we describe the identity type for two terms $M, N$ of type $S$ ? Roughly, as follows: the identity type of two terms of a $\Sigma$-type is given by the $\Sigma$-type of identities between those two terms. In more detail, given two terms

$$
\langle a, p\rangle,\langle b, q\rangle: \sum_{x: A} B(x)
$$

we have that

$$
(\langle a, p\rangle=\langle b, q\rangle) \cong \sum_{\pi: a={ }_{A} b}\left(\pi_{*}(p)={ }_{B(p)} q\right)
$$

In other words, identities between terms $\langle a, p\rangle$ and $\langle b, q\rangle$ are equivalent to identities between each of their components. But in order for this statement to make sense grammatically (i.e. be "well-typed") we need to transfer the information appropriately along the previously given identities, which is what the symbol $\pi_{*}$ indicates. Univalence then licenses us to replace "=" with " $\simeq$ " in the formula above. Therefore, the identity type of $O_{\mathrm{UF}}$ will coincide with the type of homotopy equivalences between each component. For any given $O_{\text {UF }}$ we will denote this type of "component-wise isomorphisms" by $\cong_{O}$.

As an illustration of how $\cong_{O}$ is defined, consider the case of graphs. Preformally, a graph $G=\langle V, E\rangle$ is a collection of vertices $V$ together with, for any two nodes, a collection of (possibly multiple) edges $E$ between any two vertices $v_{1}$ and $v_{2}$. We consider two such graphs $G_{1}, G_{2}$ equivalent if there is an isomorphism between $V_{1}$ and $V_{2}$ that preserves the number of edges. In UF, we can formalize graphs as the following type

$$
O_{\text {graph }} \equiv \sum_{V: \mathcal{U}} V \rightarrow V \rightarrow \text { Set }_{\mathcal{U}}
$$

Now, take two terms of this type, $\left\langle V_{1}, E_{1}\right\rangle$ and $\left\langle V_{2}, E_{2}\right\rangle$. We have:

$$
\left(\left\langle V_{1}, E_{1}\right\rangle=\left\langle V_{2}, E_{2}\right\rangle\right) \cong \sum_{p: V_{1}=V_{2}} p_{*}\left(E_{1}\right)=E_{2}
$$

As explained above, we now define $\cong_{O_{\text {graph }}}$ by replacing the identity signs on the right-hand side above by the types of of homotopy equivalences, denoted here by " $\simeq$ ". So in the example this gives us

$$
\left(\left\langle V_{1}, E_{1}\right\rangle \cong_{O_{\text {graph }}}\left\langle V_{2}, E_{2}\right\rangle\right) \equiv_{\mathrm{df}} \sum_{p: V_{1} \simeq V_{2}} p_{*}\left(E_{1}\right) \simeq E_{2}
$$

As is hopefully clear, $\cong_{O_{\text {graph }}}$ is defined canonically from $O_{\text {graph }}$, namely by the two-step process of writing out the identity type and then replacing "="

\footnotetext{
${ }^{34} S$ is of course itself a type, possibly in a higher universe of types.
} 
with " $\simeq$ ". 35

It might now appear that the relevant question to ask is whether $\cong_{O}$ captures the meaning of our original informal notion $\sim_{\text {inf }}$, whatever that may be. In one sense, this question is impossible to settle. There will always be room for a persistent skeptic to doubt that this has been achieved: "Has the meaning of group isomorphism as mathematicians understand it really been captured by the appropriate instance of homotopy equivalence in UF?" But as Quine long ago made vivid, this kind of skepticism is an artefact of the very process of translating (or, in our case, the process of formalizing) rather than of the particular language we are translating into (in our case, HoTT). And in another sense, the question has a trivial answer. After all, the identity types in UF only "see" those features of the terms being considered that were used to define these terms to begin with. And if we agree that the terms of $O_{\mathrm{UF}}$ adequately capture the features of objects of the class $O_{\text {inf }}$ that we are interested in (e.g. that they are sets, that they have a multiplicative operation etc.) then the identity type will "see" all those features and thus preserve them. And therefore, by univalence, so will $\cong_{O}$.

Therefore, the issue is not really about comparing the meaning of $\cong_{O}$ and $\sim_{\text {inf. }}$. The right question to ask, rather, is the following: $\mathrm{Is}_{O} \cong_{O}$ always deduced from $\sim_{\text {inf }}$ in a canonical way? In cases of set-level structures (e.g. groups, rings) formalized as $h$-sets-with-structure the "Structure Identity Principle" (cf. [48], Chapter 9.8 and especially Definition 9.8.4) already provides a standard way of deriving their criteria of identity from their definition, in much the same way that elementary equivalence of models in first-order logic can be derived from the signature of the corresponding first-order theory. And in those cases, the given criterion of identity does in fact coincide with $\cong_{O}$. But is there such a general method even for more complicated - and not necessarily "set-level" - structures? This is the task I now take up.

\footnotetext{
${ }^{35}$ Occasionally (e.g. in the case of (pre)categories, see below) we might be interested in more explicit descriptions of the right hand side. For example, in the case of graphs, using function extensionality and the fact that the $E_{i}$ are set-valued functions, we can obtain

$$
\left(\left\langle V_{1}, E_{1}\right\rangle \cong O_{\text {graph }}\left\langle V_{2}, E_{2}\right\rangle\right) \simeq \sum_{p: V_{1} \simeq V_{2}}\left(\prod_{x, y: V_{1}} E_{1}(x, y) \simeq E_{2}\left(p_{*}(x), p_{*}(y)\right)\right)
$$

which more closely resembles what we have come to expect graph-isomorphism to mean in practice, namely an isomorphism of the vertex-set that induces isomorphisms on the corresponding edge-sets. Nevertheless, even if the process of "rewriting conveniently" is not canonical, the process of obtaining $\cong_{O}$ is, in fact, canonical.
} 


\subsection{The general method for (CI)}

To illustrate the task at hand, as well as sketch my proposed method, let us consider the case of category theory. First, let me quickly review how category theory is formalized in UF. A widely accepted definition of a category in UF is the following:

Definition 5.1 ([1,48]). A univalent category $\mathcal{C}$ consists of the following data:

1. A type $C: \mathcal{U}$ ("objects")

2. A dependent type $\operatorname{Hom}_{\mathcal{C}}: C \rightarrow C \rightarrow \operatorname{Set}_{\mathcal{U}}$ ("Hom-sets")

3. A term 1: $\prod_{a: C} \operatorname{Hom}_{\mathcal{C}}(a, a)$ ("identity")

4. A term o: $\prod_{a, b, c: C} \operatorname{Hom}_{\mathcal{C}}(a, b) \rightarrow \operatorname{Hom}_{\mathcal{C}}(b, c) \rightarrow \operatorname{Hom}_{\mathcal{C}}(a, c)$ ("composition")

5. A term

$$
\text { assoc: } \prod_{a, b, c, d: C} \prod_{\substack{f: \operatorname{Hom}_{\mathcal{C}}(a, b) \\ g: \operatorname{Hom}_{\mathcal{C}}(b, c) \\ h: \operatorname{Hom}_{\mathcal{C}}(c, d)}} h \circ(g \circ f)=(h \circ g) \circ f
$$

which witnesses (strict) associativity.

6. A term

$$
\text { ident }: \prod_{a, b: C f: \operatorname{Hom}_{\mathcal{C}}(a, b)}\left(f \circ 1_{a}=f\right) \times\left(1_{b} \circ f=f\right)
$$

which witnesses right and left cancellability of identity maps.

7. The canonical map idtoiso $a, b: a=b \rightarrow a \cong b$ is an equivalence for all $a, b$, i.e. there exists a term

$$
\text { cat: } \left.\prod_{a, b: C} \text { isequiv(idtoiso } \text { is,b }_{a}\right)
$$

The data in (1)-(6) express in type-theoretic notation exactly the standard defining structure and properties of a category, i.e. that it consists of a collection of objects (1), a collection of arrows (2), an identity arrow on each object (3) and a composition operation (4) that is associative (5) and for which the identity arrows are left and right inverses (6). Condition (7), on 
the other hand, is new in UF ${ }^{36}$ It expresses, as we shall see, the requirement that isomorphism between objects inside a category be equivalent to identity between those objects. We will refer to it as the saturation condition.

On the other hand, there is another, more "naïve", notion that could serve as a formalization of category theory in UF:

Definition 5.2. A precategory $\mathcal{C}$ consists of data (1)-(6) in Definition 5.1.

Precategories and (univalent) categories are not equivalent structures or theories. ${ }^{37}$ So it appears that there are two distinct plausible formalizations of category theory in UF. Each of them comes with its own criterion of identity and prima facie each of these criteria of identity are plausible formalizations for categorical equivalence as it is understood in mathematical practice. So we now appear to be in a bind: if there are two inequivalent, but equally plausible, formalizations of category theory in UF then how could $\cong_{O}$ be deduced in a canonical way from $O_{\mathrm{UF}}$, given that we cannot even pick the correct " $O_{\mathrm{UF}}$ "?

What I want to say now is that we are in a bind only insofar as we assume that both univalent categories and precategories are distinct formalizations of the same informal mathematical notion. It is this dubious assumption which we must now lay to rest. Precategories and univalent categories, I submit, are best understood as (distinct) formalizations of distinct notions. Strange as this may seem from a set-theoretic point of view, it is in fact consistent with one of the fundamental tenets of UF, namely that (higher) categories are structures on (higher) groupoids rather than groupoids being categories with an extra property (all arrows are invertible). From this point of view, it is entirely natural that there should be "two (or possibly more) category theories" since a category is no longer a fundamental thing that we can only define in one way, but rather one of many possible structures that we can impose on (higher) groupoids. By analogy, consider the many kinds of orderings we have on bare sets: partial orderings, total orderings, well-orderings etc. Each of these can be studied in set theory as a separate notion. They

\footnotetext{
${ }^{36}$ Although a similar kind of condition was considered already by Hofmann and Streicher in their groupoid model for MLTT, cf. [18].

${ }^{37}$ But there is also a strong relation between them: roughly, every precategory gives rise to a univalent category (called its Rezk completion in [48]) that is "weakly equivalent" to it. For the categorically-minded reader: the obvious forgetful "functor" from univalent categories to precategories has a "left adjoint". Perhaps someone may wish to claim that this relation between the two notions shows that there is a kind of equivalence between them. This would challenge the points I go on to make below. But this is the wrong conclusion to draw. Similar relations ("forgetful-free adjunctions") are borne by classes of structures that on no reasonable account of "equivalence" should we wish to call equivalent. For example, groups and (bare) sets bear this relation.
} 
formalize different kinds of orderings that we may consider in mathematical practice. Would we want to criticize set theory for being unable to capture the "theory of orders"? No, because in set-theoretic foundations sets are fundamental and ordered sets are non-fundamental structures we define on sets. Similarly, in univalent foundations, homotopy types are fundamental and categories are non-fundamental structures we define on homotopy types. ${ }^{38}$

What is now left to show is that $\cong_{O_{\text {catiso }}}$ and $\cong_{O_{\text {catequiv }}}$ are derived from $\sim_{\text {catiso }}$ and $\sim_{\text {catequiv }}$ in a canonical way (that applies equally well to both). To see this, we must somehow describe categories-up-to-equivalence and categories-up-to-isomorphism as made up of components in such a way that both $\sim_{\text {catiso }}$ and $\sim_{\text {catequiv }}$ can be understood as asserting a component-wise isomorphism. In the case of categories-up-to-isomorphism, this is immediate since an isomorphism of categories is defined as a functor that is an isomorphism on both objects and arrows. In the case of categories-up-toequivalence the situation is more difficult. Here an equivalence of categories is usually defined as a functor that is an isomorphism on arrows but only an isomorphism-up-to-isomorphism (i.e. only essentially surjective) on objects. But such a functor, I submit, can also be understood as an abbreviated way of asking for a component-wise isomorphism if we take seriously the fact that we only care about objects in a category-up-to-equivalence up to isomorphism. In other words, the object part of a functor witnessing an equivalence between categories should be understood as providing an isomorphism of objects-upto-isomorphism, rather than an isomorphism-up-to-isomorphism of objects. And if we see it this way, then the informal criterion of identity $\sim_{\text {catequiv }}$ for categories-up-to-equivalence is of exactly the same kind as the criterion of identity $\sim_{\text {catiso }}$ for categories-up-to-isomorphism.

As a result, if we formalize $O_{\text {catiso }}$ as the type of precategories and $O_{\text {catequiv }}$ as the type of univalent categories, then we can argue that $\cong_{O_{\text {catiso }}}$ and $\cong_{O_{\text {catequiv }}}$ are canonically obtained from $\sim_{\text {catiso }}$ and $\sim_{\text {catequiv }}$ for the exact same reasons. Indeed this point of view correlates very well with the formalization of category theory in [48]: isomorphism of precategories (Definition 9.4 .8 ) is easily seen to be equivalent to $\cong{ }_{O_{\text {catiso }}}$ (Lemma 9.4.14, essentially) and the definition can be repeated in the case of univalent categories. Now it so happens that because of the extra saturation condition, $\cong_{O_{\text {catequiv }}}$ in the case of univalent categories is also itself equivalent to a relation that more closely resembles what usually goes under the name of "equivalence of categories" in mathematical practice (Lemma 9.4.14). But that is beside the

\footnotetext{
${ }^{38}$ Accepting the distinction between categories up to isomorphism and categories up to equivalence depends on some level on accepting that groupoids are more fundamental than categories. Although it is by no means a trivial or obvious thesis, I will say no more about it here.
} 
point here. What matters, rather, is that both isomorphism of precategories and "equivalence" of univalent categories can be obtained, up to equivalence, as component-wise isomorphisms in exactly the same way. ${ }^{39}$

The above discussion illustrates very well the general method of formalizing criteria of identity as component-wise isomorphisms where, as ever, we understand "isomorphism" as a placeholder for the notion of homotopy equivalence. It is now time we stated this general method. For a given theoretical context given by $O_{\text {inf }}$ and $\sim_{\text {inf }}$ it goes as follows:

1. Decomposition: break down the structures of $O_{\text {inf }}$ into all their relevant components $\left(S_{1}, \ldots, S_{n}\right)$, some of which may depend on others.

2. Component-wise isomorphism: determine the relevant "local" criteria of identity $\left(\sim_{1}, \ldots, \sim_{n}\right)$ for objects in each of these components in such a way that the "global" criterion of identity $\sim_{\text {inf }}$ can be understood as a component-wise isomorphism.

3. Saturation: formalize $O_{\text {inf }}$ in such a way that the formalized "local" criteria of identity $\sim_{i}$ for each formalized component $S_{i}$ are homotopy equivalent to the identity type.

4. Invariance: Argue that $\cong_{O}$ is the correct formalization of $\sim_{\text {inf }}$.

To illustrate how this general method works it is helpful to sketch how when applied to the theoretical context of categories-up-to-equivalence it gives us the formalized notion of univalent categories in HoTT:

1. $O_{\text {inf }}$ is given by structures with the following components: Objects $(O b)$, Arrows $(A)$, Identities $(I)$ and Composition (o). These satisfy the usual properties that define a category.

2. The criterion of identity $\sim_{O b}$ for objects is isomorphism defined in the usual way in terms of $A, I$ and $\circ$. The criteria of identity for all the rest of the components of the structure are standard. Categorical equivalence can then be understood as a component-wise isomorphism as sketched above.

3. We formalize $O_{\text {inf }}$ as the type of univalent categories $O_{\text {catequiv }}$, where condition (7) provides the required saturation condition between the formal version of $\sim_{O b}$ and the identity type of $O b$.

\footnotetext{
${ }^{39} \mathrm{An}$ anonymous referee has correctly pointed out that the notion of a strict category ([48], Definition 9.6.1) which is a precategory where the type of objects is an $h$-set is perhaps a better formalization of the informal notion of category-up-to-isomorphism. So it is worth noting that everything I have said so far could be repeated for strict categories instead of precategories, changing nothing of substance.
} 
4. As argued above, $\cong O_{\text {catequiv }}$ is then the correct formalization of $\sim_{\text {inf. }}$.

Needless to say, the above-described general method is not a formal algorithm. It is meant, rather, as a general rule of thumb about how to produce invariant formalizations of theoretical contexts in UF. As a rule of thumb, it will require specific tweaks in specific cases and of course will be subject to varying interpretations. I will make no claim here that this method is precise enough to pin down a unique formalization of every theoretical context. But it is, I submit, precise enough to establish a general approach to invariant formalizations - and no more than that is required as an argument in support of (CI).

With this qualification in mind, we must now ask the much more difficult question: does the method for determining criteria of identity as componentwise isomorphisms extend to most structures of interest in mathematical practice? Moreover, does it produce formalizations that mathematicians would find native? To this, and more, the next section is devoted.

\section{Formalizing all of mathematics in HoTT}

To satisfy criterion (F), HoTT must at the very least prove capable of encoding all of mathematics as it is currently being practiced, i.e. it needs to be a "big-f" Foundation for all of mathematics rather than a "small-f" foundation for a specific mathematical discipline. Therefore, I will begin my argument for (F) by countering the three main objections that have been put forward against UF's capacity as a "big-f" Foundation. Then I will argue that the general method outlined in Section 5 can be used to formalize most theoretical contexts and that, in addition, the way in which this is achieved would not appear alien to practicing mathematicians.

\subsection{Is HoTT a "big-f" Foundation for all of mathemat- ics?}

As a formalization of the Univalent Foundations, HoTT is based on an extension of a well-understood formal system (intensional MLTT) that itself has been plausibly discussed as offering a self-sufficient foundation for all of mathematics. As such, the question of whether or not HoTT can be regarded as a "big-f" Foundation for all of mathematics seems to me - if not unfair then at least to be one in which the burden of proof lies firmly on the side of the skeptic. Therefore, I will not provide a positive account for an affirmative answer to the title question, but content myself with countering what I take to be the most widespread objections to such an affirmative answer. 


\section{(A) "HoTT cannot be a foundation for all of mathematics because not all of mathematics is homotopy theory!"}

Response to (A): This objection (the most common one) betrays a basic misunderstanding about the distinction between the analytic and the synthetic aspect of UF. UF certainly contains a synthetic theory of $\infty$ groupoids ${ }^{40}$ and such a theory is certainly about (abstract) homotopy theory. But UF also contains an "analytic" (for lack of a better term) version of set theory, namely that given by $h$-sets (i.e. 0-types). Therefore, the short and sweet response to (A) is simply this: UF is homotopy theory only insofar as it constitutes a synthetic theory of $\infty$-groupoids. But UF is not merely a synthetic theory of $\infty$-groupoids. And it is exactly all its other features (including its "analytic" set theory) that make it a viable candidate to serve as a (structuralist) Foundation of mathematics. To put the point differently: UF is not merely a theory about homotopy types; it is a theory allowing you to carry out constructions on homotopy types. ${ }^{41}$

Yet even though (A) fails as an objection, it does still highlight an important issue: the synthetic and the "analytic" side of UF (formalized as HoTT) do not necessarily interact as one would expect. Let me illustrate with an example. Suppose I am interested in proving that the topological circle $S^{1}$ is not homeomorphic to the topological sphere $S^{2}$. I then define $S^{1}$ and $S^{2}$ synthetically as higher inductive types (call them "synthetic $S^{1}$ " and "synthetic $S^{2}$ ") and then calculate their fundamental groups in the now wellknown way outlined in [48]. Have I thereby proved that topological $S^{1}$ is not homeomorphic to topological $S^{2}$ ? Inside HoTT, I have not. ${ }^{42}$ Which leaves the obvious worry: if in order to draw conclusions about spaces up to homeomorphism you then have to repeat the relevant proofs (e.g. of $\pi_{1}\left(S^{1}\right)=\mathbb{Z}$ ) at the level of $h$-sets, then how much is the synthetic side of UF adding to UF-regarded-as-a-foundation?

I think this is an important limitation of HoTT but no deal-breaker with respect to UF's capacity to serve as a foundation for mathematics, for two

\footnotetext{
${ }^{40}$ For a precise and thorough explanation of the way in which HoTT/UF is a synthetic theory of $\infty$-groupoids see Shulman [45].

${ }^{41}$ It is important to note that among such constructions are also those of homotopy types that can be understood as models of set theory as traditionally conceived. For example, the type $\operatorname{Set}_{\mathcal{U}}$ of $h$-sets without any further assumptions on HoTT is a model of a weak predicative set theory (a " $\Pi W$-pretopos") and if we assume LEM then a model of ZFC can also be constructed as a higher inductive type (cf. [48], 10.5).

${ }^{42}$ This is because it does not even make sense to consider synthetic $S^{1}$ and $S^{2}$ "up to homeomorphism". The very statement " $S^{1}$ is not homeomorphic to $S^{2}$ " cannot be stated in UF-regarded-as-a-synthetic-theory-of- $\infty$-groupoids. It must be stated with $S^{1}$ and $S^{2}$ defined topologically, i.e. as $h$-sets with appropriate extra properties and structure mirroring the way in which these spaces are usually defined in point-set topology.
} 
reasons. Firstly, the fact that synthetic calculations of homotopy groups in HoTT cannot be used "as is" to formalize traditional inferences we might draw about spaces up to homeomorphism does not mean that these synthetic calculations are devoid of mathematical merit. Nor does it mean that the above-described inferences (from the non-isomorphism of fundamental groups to the non-homeomorphism of spaces) are not formalizable at all: they can still very well be formalized at the level of $h$-sets (by repeating the classical proofs). Secondly, it is worth remarking that there could be possible extensions of HoTT that could ameliorate this situation. Most prominently, there is a version of HoTT called cohesive HoTT (cf. [41,42,46]) that makes the interaction between the synthetic and the "analytic" side more robust, essentially by adding a kind of "topological" (or "cohesive") structure to bare homotopy types. This extra structure allows one to recover the "analytic" versions of mathematical structures from their synthetic versions. In particular, one can prove (cf. [46]) that the realization of the synthetic $S^{1}$ is equivalent to the topological circle, thus solving the problem described above. Perhaps this means that an argument could be made that cohesive HoTT - even though motivated by questions in the foundations of higher gauge theories in physics - could actually provide a worthy alternative "foundational" formalization of UF. At the very least this demonstrates that the lack of interaction between the analytic and the synthetic side in the current formulation of HoTT is not an insurmountable obstruction.

(B) "UF cannot be a Foundation for mathematics because its basic objects are not fundamental. $\infty$-groupoids were initially defined analytically within set theory as sets with extra structure and properties. Therefore, sets are more fundamental than $\infty$-groupoids."

Response to (B): The objection here is that a synthetic theory of a certain type of structure (e.g. $\infty$-groupoids in the case of UF) cannot be a Foundation for the whole of mathematics if that type of structure can be defined analytically in another formal system (e.g. $\infty$-groupoids as Kan simplicial sets in ZFC). But this argument can just as easily be turned on its head: by the same token one can argue that since UF provides an analytic description of a set (as an $h$-set) then set theory cannot be a Foundation since its basic objects can be defined analytically in another formal system (as $h$-sets in UF).

The debate will then come down to which type of structure is more "intuitively" fundamental: "shapes" or "collections". But this no longer has anything to do with (B). Furthermore, being able to define $X$ in terms of $Y$ seems to me a highly unreliable guide to $X$ or $Y$ 's relative fundamentality: 
we can define lines in terms of points (e.g. in Cartesian geometry) but we can also define points in terms of lines (e.g. as the intersections of lines) and these facts do not seem at all relevant in deciding whether points or lines are more fundamental. As for historical reasons of priority ("sets are more fundamental than $\infty$-groupoids because they were the first to be thought of as the basic objects of mathematics") I can only say that the correlation of historical precedence and fundamentality is tenuous at best: would we similarly want to say that point-shaped particles are surely more fundamental than strings because point-shaped particles were the first, historically, to be thought of as the fundamental constituents of matter?

Nevertheless, beyond relative fundamentality, one can also question the inherent simplicity of homotopy types. In other words, are homotopy types objectively simple enough to serve as the basic entities of a foundation of mathematics? I believe the way to answer this is to ask the same question of the criterion of identity which defines homotopy types, viz. homotopy equivalence. For the simplicity of a certain concept is surely positively correlated to the simplicity of its identity conditions. Now a homotopy equivalence is simply a continuous ("smooth", though not in the sense of calculus) deformation of one space into another. To formalize this idea takes a lot of effort, especially in set theory, but this does not detract from its conceptual simplicity. And since homotopy types are supposed to be exactly what gets preserved by homotopy equivalence, they share in the simplicity of the notion of continuous deformation. Quite simply, homotopy types are what you cannot change about a space any way you continuously deform it. ${ }^{43}$ And this way of describing what they are is good evidence that they possess some inherent simplicity that makes them suitable to play the role of the basic objects of a foundation of mathematics.

(C) "HoTT is merely a new kind of semantics for a pre-existing formal system. It was not purpose-built as a formal system in order to capture a pre-formal semantics in the same way that e.g. $Z F(C)$ was purposebuilt to capture a pre-formal understanding of the cumulative hierarchy of sets."

Response to (C): It is true that HoTT builds upon a pre-existing formal system (intensional MLTT) that originally had nothing to do with homotopy theory. But the recycling and repurposing of formal systems is not

\footnotetext{
${ }^{43}$ Marquis makes a very similar point in arguing for the fundamentality of homotopy types: "I submit that the notion of [homotopy equivalence] at work here is philosophically fundamental: we are dealing with entities that can be continuously transformed into one another." ([30], p. 2151)
} 
only technically advantageous but also natural and unavoidable. Therefore, on pragmatic grounds, the fact that the original "meaning explanation" of Martin-Löf in [31] has nothing to do with the homotopic semantics of HoTT should not be seen as problematic. First-order predicate logic can also be seen as a repurposing of Frege's calculus in the Begriffsschrift, but surely we wouldn't want to claim that this presents conceptual worries for ZFC simply because of their differing intended semantics. Furthermore, in the specific case of intensional MLTT the higher structure of identity types was for a long time considered a kind of aesthetic flaw of the system since noone could make sense of it semantically or find any use for it syntactically. Indeed, until Hofmann and Streicher [18] constructed the groupoid model of MLTT it was considered plausible that the UIP axiom (which would collapse the higher structure of types and is incompatible with univalence) was provable in MLTT. Through the homotopy interpretation, this higher structure of identity types is once and for all revealed as a feature rather than a bug of intensional MLTT. And this, to my mind, suffices to quell any worries that intensional MLTT is being somehow misappropriated by UF.

\subsection{Native formalizations for all theoretical contexts?}

In Section 5 I argued for (CI) by describing a general method for formalizing criteria of identity for mathematical structures as homotopy equivalences in UF. By virtue of this general description, this method is uniform in the sense of Section 3: wherever it applies, it applies for the same reason, i.e. for the reason that it encodes criteria of identity as componentwise isomorphisms. In addition to the objections considered above we must now ask whether the method is wide enough: does it plausibly cover all structures of mathematical interest? We must also ask whether it is native to mathematicians: does it yield formalized notions comprehensible to mathematicians familiar exclusively with their informal counterparts?

Let me begin with the first question, regarding the scope of the general method. There are two approaches one could take here, one formal, one informal. The formal approach would consist of providing a general notion of a signature, plausibly wide enough to cover all structures of mathematical interest, for which we would then prove a result analogous to the Structure Identity Principle in [48]. I believe it is possible to define such a general notion of a signature and have developed what I think is a plausible candidate in [47]. Proving a Structure Identity Principle in terms of this general notion of signature is certainly conceivable. However, I do not think that establishing (SFOM) ought to rely on such a formal result. I understand (SFOM) here as an informal thesis, gaining in generality what it perhaps loses in formal 
rigour. This is why I think it suffices for the purposes of this paper to rely solely on an informal argument for $(\mathrm{F})$.

This informal argument would need to establish that most theoretical contexts in the practice of mathematics can be formalized using the general method outlined in Section 5.2. To do so, first note that informal criteria of identity will always strive to preserve all and only the structure of the objects under consideration. Insofar as we are clear on what that structure is, then there should be a clear and standard way to list the components that are relevant to the objects under consideration. For example, if we are interested in categories with specified limits, then we know that we should be able to separate their objects up to equality and not merely up to isomorphism. Second, as long as we are able to list these components, then we are able to formalize them inside UF, at least insofar as my responses to the objections in Section 6.1 hold up. This will give us a formal definition of $O_{\mathrm{UF}}$ in HoTT. And then, finally, if we follow the method of Section 5 the correct criterion of identity will be phrased in terms of isomorphisms between these (formalized) components and will thus coincide with $\cong_{O}$. Therefore, insofar as a mathematical structure of interest can be broken down in components (each of which may come equipped with its own "local" criterion of identity) then the class of such structures can be invariantly formalized according to our general method.

However, this simple-sounding vision will face many difficult cases. Firstly, with respect to the "small" theoretical contexts like the natural numbers that are best formalized as individual types, we might find several examples in UF that strain the principle of regarding identities in them as degenerate versions of homotopy equivalence. An important such case is the synthetic formalization of homotopy types, e.g. of the circle $S^{1}$ as a higher inductive type. It is admittedly unclear how my method can make sense of such a formalization. In my defense, there is a blurry line between the role of higher inductive types as formalizations of mathematical notions and their role as actual mathematical structures themselves. This line is likely to remain blurry until a general specification for higher inductive types is agreed upon. Until such time comes it is difficult to say much about how problematic such cases really will turn out to be for (SFOM).

Secondly, HoTT currently faces difficulties in formalizing notions from higher category theory (paradigmatically, those developed by Lurie in [24] and related work). In particular, somewhat ironically, HoTT is not very good at formalizing itself. Due to issues related to being able to express infinite socalled coherence conditions, the standard versions of HoTT (e.g. the one in [48]) are not able to express what it would be for a type (e.g. a universe $\mathcal{U}$ ) to 
be a model of HoTT. ${ }^{44}$ Similar problems arise for other such " $\infty$-structures". There is therefore a likelihood that as more of these structures find their way into the practice of mathematics, the less well HoTT will be able to live up to (SFOM). I think this is a serious issue to consider, but it is not one that an appropriately designed HoTT could not fix. Indeed, there exist solutions that have already been proposed (e.g. Voevodsky's HTS) but they remain underdeveloped. But in any case, it must be conceded that until some general consensus emerges as to what kind of HoTTs will prove equal to the task, such theoretical contexts involving $\infty$-structures will present difficult cases.

Thirdly, with respect to the "large" theoretical contexts like category theory, one may worry that my general method works only because of some especially nice algebraic properties of (higher) categories and that it will break down in "less algebraic" theoretical contexts. Take for example as our theoretical context topological spaces up to homotopy understood in the traditional point-set-topological way - for ease of reference I will call the objects traditional topological spaces and their criterion of identity traditional homotopy equivalence. In the manner outlined by my general method, we may now try to understand a traditional homotopy equivalence as a componentwise notion if we agree that the correct criterion of identity for points in a space $X$ is for them to be connected by a continuous path. Namely, for a given topological space $X$ and two points $x, x^{\prime}$ in $X$ we define $x \sim_{\text {inf }} x^{\prime}$ as the class of continuous paths between $x$ and $x^{\prime}$. But now we are in a situation where the topology $\mathcal{T}$ of the space $X$ will not in general respect $\sim_{\text {inf }}$ since it is quite possible that there is an open set $U \in \mathcal{T}$ such that $x \in U, x^{\prime} \notin U$ but $x \sim_{\text {inf }} x^{\prime}$. Clearly, in such a situation we will have to violate either step (1) or step (3) of the general method. For we will either have to sacrifice talking about open sets, thus ignoring one component of the notion in question, or fail to assert that the (formalized) notion of continuous path can be equivalent to identity. (If it were, then any two points connected by a path would have to be contained in the same open sets, something which does not hold informally. $)^{45}$

What I want to say in response to such cases is summed up as follows: if the intended criterion of identity for one component $S_{1}$ of a structure is not respected by some other component $S_{2}$ (that may depend on $S_{1}$ ) then it is not a criterion of identity. To elaborate, it is helpful to consider a toy case. So consider a theoretical context where structures in $O_{\text {inf }}$ are given by (at least) two components $X$ and $S$ and we impose some criterion of identity

\footnotetext{
${ }^{44}$ For a precise description to the problem, as well as an illuminating introduction to possible methods that could solve it, cf. [44].

${ }^{45}$ The case of traditional topological spaces as a challenge to my general method was raised by an anonymous referee, to whom I clearly owe this entire discussion.
} 
$\sim_{X}$ on $X$. A formalization of this set-up in UF is given by the following data (where we use the same notation for $X$ and $S$ as their informal counterparts):

$$
\begin{aligned}
& X: \mathcal{U} \\
& S: X \rightarrow \mathcal{U} \\
& \sim: X \rightarrow X \rightarrow \mathcal{U}
\end{aligned}
$$

In the example of traditional topological spaces, one can think of $S$ as a propositional family expressing whether a given term is in a certain open set and $x \sim y$ as the type continuous paths between any two terms $x, y: X$ (where "continuous path" is defined in terms of some notion of topology in terms of which $S$ is also defined).

Now, on the one hand, it may be the case that we have two terms in one component related by the criterion of identity in that component that are explicitly separated by another component. In terms of the toy example, this means that we have $a, b: X$ such that there is $f: a \sim b$ but also $S(a)$ and $\neg S(b) .{ }^{46}$ I think it is clear that if one is considering a structure that contains certain objects that are separated by one component $S$ of that structure, then no relation $\sim$ defined in terms of any other component that relates objects that $S$ separates can properly be called a criterion of identity. Any criterion of identity deserving of the name must be one that respects any other component of the structure under consideration. This indicates, for example, that we should not formalize traditional topological spaces up to traditional homotopy equivalence in HoTT as $h$-sets together with a family of "open" subtypes satisfying the required conditions. For the fact that we can define continuous paths in terms of open sets in mathematical practice should not make us forever beholden to these open sets just as the fact that pre-formally we define isomorphism in a category by separating objects up to equality shouldn't mean we are forever beholden to equality of objects.

On the other hand, there may not necessarily be any component "disrespecting" a proposed criterion of identity for another component, but that criterion of identity may be too "loose" to induce equivalences between the rest of the components of the structure. For, ideally, we should want that if $a \sim b$ then for any other component $S$ that depends on $a$ or $b$ we have $S(a) \cong S(b)$ (where $\cong$ is the preferred criterion of identity for entities of the kind that $\mathrm{S}$ takes values in). For example, this is true in the case of categories because an isomorphism between two objects also induces a bijection between corresponding hom-sets. ${ }^{47}$ Yet we are perfectly well-able to imagine criteria of identity that do not induce equivalences on the rest of the components

\footnotetext{
${ }^{46}$ Here $\neg S(b)$ can be thought of as notation for $S(b) \rightarrow 0$.

${ }^{47}$ More precisely, for any other object $c$ an isomorphism $f: a \cong b$ induces a bijec-
} 
of the structure but for which it is not contradictory to assert a saturation axiom in the style of univalent categories. But this is not something that should trouble us: for by asserting a saturation axiom we do ensure that all other components of our structure end up respecting our chosen criterion of identity in the way desired - and without any loss of information. ${ }^{48}$

The main difficulty shown up by the case of traditional topological spaces is that it is often unclear in mathematical practice how to "remove" certain pieces of structure in terms of which criteria of identity are expressed. For example, in the case of categories-up-to-equivalence we might as well "remove" the notion of equality of objects once we have decided that the criterion of identity for objects is given by isomorphism. This is easily done in this case since none of the already-given components (arrows, identities, composition) separate objects more finely than isomorphism. By contrast, there is no easy way to "remove" equality of points in a traditional topological space in favour of continuous paths between them since open sets may very well separate two such points. Nor is there any straightforward way to simply "remove" open sets since the very notion of a continuous path is defined in terms of them.

On the other hand, it is far from clear that the intuitive notion of a continuous path between points must be expressed this way, i.e. in terms of open sets that already separate distinct points. Indeed, the field of abstract homotopy theory is largely devoted to setting up the theoretical context of "topological spaces up to homotopy" in such a way that this does not occur, i.e. by "algebraicizing" them in a useful way. But it is unclear whether this process of "algebraicizing" can always take place - and even less clear that it can always result in a theoretical context that practicing mathematicians would find practically indistinguishable from the one we began with. Nevertheless, it seems to me that such difficult cases usually arise because of an inevitable bias towards thinking of the objects of mathematics in terms of sets, which thus tends to force us into making very many distinctions that might not prove necessary to faithfully formalizing the structures of interest. The example of traditional topological spaces is a case in point: although the point-set definition of a topological space makes perfect sense and can be

tion $f^{*}: \operatorname{Hom}(c, a) \cong \operatorname{Hom}(c, b)$ by transporting morphisms along $f$ and similarly for $\operatorname{Hom}(-, c)$.

${ }^{48}$ What the above-described situation certainly does prevent us from doing is a Rezkcompletion-style construction. The reason that Rezk completion works in the case of categories is exactly because we can use a given isomorphism to induce a bijection (i.e. $h$ equivalence) between hom-sets. This means that isomorphisms "already" respect hom-sets. But we have no reason to expect this to be the case in general. So the Rezk completion construction certainly does not generalize to arbitrary structures, as of course one ought to expect. 
studied as a mathematical object in its own right, it is by no account forced upon us when we want to study spaces up to traditional homotopy. ${ }^{49}$

Even so, the second question remains: are the formalized notions we end up with native ones? Will they be comprehensible to the practicing mathematician (insofar as any formal system is)? In one sense, the idea of formalizing anything as a structure on a homotopy type is likely to confuse practicing mathematicians far more than the idea of formalizing such structures in terms of sets. But this is a premature criticism at least insofar as it depends on the novelty of formalizing mathematical objects in terms of homotopy types. It is certainly an idea that no-one had taken seriously pre-UF and which at this point still may appear mysterious. In his recent talk [52] Voevodsky emphasizes exactly this point:

The third component [of any foundational system] is a structure that enables humans to encode mathematical ideas in terms of the objects directly associated with [the structures that provide the semantics for the sentences of this language in terms of mental objects intuitively comprehensible to humans. In UF this third component], a way to encode general mathematical notions in terms of homotopy types, is based on the reversal of Grothendieck's ideas considered in [[20]].

Both mathematically and philosophically, this is the deepest and least understood part of the story.

But from its novelty, it does not follow that it is an idea definitively incompatible with mathematical practice (or, at the very least, with the mathematical practice of today).

On the other hand, the idea that any structure and property can be transferred along isomorphic objects is indeed very close to contemporary

\footnotetext{
${ }^{49}$ One is inevitably reminded of Grothendieck's beautiful ruminations in his Esquisse d'un Programme [15]:

[W]hen one tries to do topological geometry in the technical context of topological spaces, one is confronted at each step with spurious difficulties related to wild phenomena. [...] This situation, like so often already in the history of our science, simply reveals the almost insurmountable inertia of the mind, burdened by a heavy weight of conditioning, which makes it difficult to take a real look at a foundational question, thus at the context in which we live, breathe, work - accepting it, rather, as immutable data. [...] It is this again which explains why the rigid framework of general topology is patiently dragged along by generation after generation of topologists for whom "wildness" is a fatal necessity, rooted in the nature of things. (pp. 258-259)
} 
mathematical thinking, as has been emphasized numerous times in the extensive literature on categorical foundations of mathematics. In that respect, the formalized notions we end up with in UF are much closer to the way of thinking of practicing mathematicians. The details of the formalization and the formal system itself are certainly at the present moment in time not as widespread as set-theoretic formalizations in terms of $\mathrm{ZF}(\mathrm{C})$. But the fundamental idea of caring about mathematical structures only up to isomorphism - vastly generalized in HoTT - is certainly a defining trait of the mathematical practice of today. And insofar as the general method outlined here depends crucially on formalizing this exact trait, then the essence of the formalizations themselves should prove native to practicing mathematicians, at least modulo the type-theoretic language barrier.

In conclusion, the general method I have offered cannot be taken as definitive. But neither, of course, is it meant to be. The process of formalization, after all, is not itself definitively formalizable. Insofar as formalizing mathematical structures and arguments still requires human intervention there will always be difficult cases, requiring patches and choices not reducible to general methods and principles. Ultimately, my argument for (F) - and therefore also for (SFOM) - can only rely on an abundance of clear-cut cases in which the general method does apply rather than on a purported clear-cut argument that all cases are covered.

\section{Conclusion}

In this paper, I have examined the extent to which UF satisfies (SFOM). What I have argued for should not (and cannot) be taken to be a once-andfor-all-argument that UF will always provide a way of formalizing mathematical structures of interest in an invariant manner. I would probably go so far as to say that the general method I have described in Section 5 does provide such a general method for formalizing most mathematical structures of current interest. But mathematical practice is changing and we may soon begin to study structures with properties that are just as difficult or unnatural to formalize in UF as higher-categorical structures in ZFC. Therefore, insofar as (SFOM) is tied to the mathematical practice of today (and of the immediate future) no claim that I make in this paper can exceed it. Nevertheless, even with this limitation in mind, there is certainly an important conclusion that emerges from the current argument: with respect to (SFOM), UF presents a quite significant improvement over (extensional or structural) set-theoretic foundations of mathematics.

Does this now mean: off with set theory's head? Not at all. In fact, I think 
UF and set theory, especially ZFC, are not best understood as competing foundations. The relationship I envisage between ZFC and UF is essentially that of successive (but co-existing) conceptual schemes in the Quinean sense. Neurath's famous (and somewhat overused) metaphor of the mariner is apt: UF is slowly rebuilding the ship of the foundations of mathematics using the material of set theory on which it still relies to stay afloat. For example, ZFC is used to build the simplicial model of UF [21] and the construction of homotopy types in ZFC still guides our intuitions about what ought to be true of them. A pluralistic picture, I believe, is the most appropriate attitude to take here - one in which set-theoretic foundations and UF establish a symbiotic relationship. I intend to spell out this picture, and explain its advantages, in future work.

\section{References}

[1] Benedikt Ahrens, Krzysztof Kapulkin, and Michael Shulman, Univalent categories and the rezk completion, Extended abstracts fall 2013, 2015, pp. 75-76.

[2] S. Awodey, An answer to Hellman's question: 'Does category theory provide a framework for mathematical structuralism?', Philosophia Mathematica 12 (1) (2004), 5464.

[3] _ Structuralism, invariance and univalence, Philosophia Mathematica 22 (1) (2014), 1-11.

[4] S. Awodey and M. A. Warren, Homotopy theoretic models of identity types, Math. Proc. Camb. Phil. Soc. 146 (2009), no. 45, 45-55.

[5] P. Benacerraf, What numbers could not be, The Philosophical Review 74 (1) (Jan. 1965), 47-73.

[6] Marc Bezem, Thierry Coquand, and Simon Huber, A model of type theory in cubical sets, 19th international conference on types for proofs and programs (types 2013), 2014, pp. 107-128.

[7] Georges Blanc, Equivalence naturelle et formules logiques en théorie des catégories, Archive for Mathematical Logic 19 (1978), no. 1, 131-137.

[8] J. Burgess, Putting structuralism in its place, 2013. Manuscript.

[9] _ Rigor and structure, Oxford University Press, Oxford, 2014.

[10] Emily Carson, Kant on the method of mathematics, Journal of the History of Philosophy 37 (1999), no. 4, 629-652.

[11] Cyril Cohen, Thierry Coquand, Simon Huber, and Anders Mörtberg, Cubical type theory: a constructive interpretation of the univalence axiom (2015). https://www. math.ias.edu/ amortberg/papers/cubicaltt.pdf.

[12] Peter Freyd, Properties invariant within equivalence types of categories, Algebra, topology, and category theory (a collection of papers in honor of Samuel Eilenberg) (1976), 55-61. 
[13] Michael Friedman, Kant's theory of geometry, The Philosophical Review 94 (1985), no. $4,455-506$.

[14] _ Kant on geometry and spatial intuition, Synthese 186 (2012), 231-255.

[15] Alexandre Grothendieck, Esquisse d'un programme, London Mathematical Society Lecture Note Series (1997), 5-48. http://www.landsburg.com/grothendieck/EsquisseEng.pdf.

[16] G. Hellman, Three varieties of mathematical structuralism, Philosophia Mathematica 9 (2001), no. 2, 184-211.

[17] J. Hintikka, Kant on the mathematical method, The Monist 51, no. 3, 352-375.

[18] M. Hofmann and T. Streicher, The groupoid interpretation of type theory 36 (1998), 83-111. MR1686862

[19] D. Hogan, Kant and the character of mathematical inference, Kant's philosophy of mathematics, 2015.

[20] M. Kapranov and V. Voevodsky, $\infty$-groupoids and homotopy types, Cahiers Topologie Géom. Différentielle Catég. 32 (1991), no. 1, 29-46. International Category Theory Meeting (Bangor, 1989 and Cambridge, 1990). MR1130401 (93c:55006)

[21] K. Kapulkin, P. Lumsdaine, and V. Voevodsky, The simplicial model of univalent foundations, arXiv:1211.2851v2 (2014).

[22] W. Lawvere, An elementary theory of the category of sets, Reprints in Theory and Applications of Categories 12 (2005), 1-35.

[23] T. Leinster, Rethinking set theory, American Mathematical Monthly 121 (2014), no. 5, 403-415, available at http://arxiv.org/abs/1212.6543.

[24] Jacob Lurie, Higher Topos Theory, Princeton University Press, 2009.

[25] M. Makkai, First order logic with dependent sorts with applications to category theory, 1995. http://www.math.mcgill.ca/makkai/folds/foldsinpdf/FOLDS.pdf.

[26] Towards a categorical foundation of mathematics: Logic colloquium '95, Lecture Notes in Logic 11 (1998), 153-190.

[27] (2013). http://www . math.mcgill.ca/makkai/Various/MateFest2013.pdf.

[28] J-P. Marquis, From a geometrical point of view: A study of the history and philosophy of category theory, Vol. 14, Springer Science \& Business Media, 2008.

[29] _ Categorical foundations of mathematics: Or how to provide foundations for abstract mathematics, The Review of Symbolic Logic 6 (March 2013), no. 1.

[30] Jean-Pierre Marquis, Mathematical forms and forms of mathematics: leaving the shores of extensional mathematics, Synthese 190 (2013), no. 12, 2141-2164.

[31] Per Martin-Löf, Intuitionistic type theory, Bibliopolis, 1984.

[32] C. McLarty, Numbers can be just what they have to, Nous 27 (1993), no. 4, 487-498.

[33] _ Exploring categorical structuralism, Philos. Math. (3) 12 (2004), no. 1, 37-53. MR2033719 (2005b:03018a) 
[34] J. Mycielski, A lattice of interpretability types of theories, The Journal of Symbolic Logic 42 (1977), no. 2, 297-305.

[35] Erik Palmgren, Constructivist and structuralist foundations: Bishop's and Lawvere's theories of sets, Annals of Pure and Applied Logic 163 (2012), no. 10, 1384-1399.

[36] C. Parsons, The structuralist view of mathematical objects, Synthese 84 (1990), 303346.

[37] Charles Parsons, Kant's philosophy of arithmetic, KantÕs philosophy of mathematics, 1992, pp. 43-79.

[38] H. Putnam, Mathematics without foundations, Philosophy of mathematics, 2nd ed.

[39] Willard Van Orman Quine, Ontological relativity and other essays, Columbia University Press, 1969.

[40] M. Resnik, Mathematics as a science of patterns: Ontology and reference, Nous 15 (1981), 529-550.

[41] U. Schreiber, Differential cohomology in a cohesive $\infty$-topos (2013), available at http: //arxiv.org/abs/1310.7930.

[42] U. Schreiber and M. Shulman, Quantum gauge field theory in cohesive homotopy type theory (2012), available at \url\{http://ncatlab.org/schreiber/files/ QFTinCohesiveHoTT.pdf\}.

[43] S. Shapiro, Philosophy of mathematics: Structure and ontology, Oxford University Press, Oxford, 1997.

[44] M. Shulman, Homotopy type theory should eat itself (but so far, it's too big to swallow).: Homotopy type theory blog post, 2014.

[45] Homotopy type theory: A synthetic approach to higher equalities, Categories for the working philosopher, 2016.

[46] Michael Shulman, Brouwer's fixed-point theorem in real-cohesive homotopy type theory, arXiv preprint arXiv:1509.07584 (2015).

[47] Dimitris Tsementzis, Homotopy model theory $i$ : Syntax and semantics, arXiv preprint arXiv:1603.03092 (2016).

[48] Univalent Foundations Program, Homotopy type theory: Univalent foundations of mathematics (2013). http://homotopytypetheory.org/book.

[49] V. Voevodsky, Foundations of mathematics and homotopy theory (2006). https://www.math.ias.edu/vladimir/sites/math.ias.edu.vladimir/files/ $\mathrm{VV} \backslash \% 20$ Slides.pdf.

[50] Univalent foundations project (2010). http://www.math.ias.edu/ vladimir/files/univalent_foundations_project.pdf.

[51] An experimental library of formalized mathematics based on univalent foundations (2014). http://arxiv.org/pdf/1401.0053.pdf.

[52] Univalent Foundations (lecture at the IAS) (2014). https: //www.math.ias. edu/vladimir/sites/math.ias.edu.vladimir/files/2014_IAS.pdf.

[53] M. A. Warren, Homotopy theoretic aspects of constructive type theory, Ph.D. Thesis, 2008. 